\title{
REVIEWS
}

Check for updates

\section{Advances in oligonucleotide drug delivery}

Thomas C. Roberts $\mathbb{1}^{1,2 凶}{\text {, Robert Langer } \mathbb{1}^{3}}^{3}$ and Matthew J. A. Wood $\mathbb{1}^{1,2 凶}$

Abstract | Oligonucleotides can be used to modulate gene expression via a range of processes

including RNAi, target degradation by RNase $\mathrm{H}$-mediated cleavage, splicing modulation, non-coding RNA inhibition, gene activation and programmed gene editing. As such, these molecules have potential therapeutic applications for myriad indications, with several oligonucleotide drugs recently gaining approval. However, despite recent technological advances, achieving efficient oligonucleotide delivery, particularly to extrahepatic tissues, remains a major translational limitation. Here, we provide an overview of oligonucleotide-based drug platforms, focusing on key approaches - including chemical modification, bioconjugation and the use of nanocarriers - which aim to address the delivery challenge.

Oligonucleotides are nucleic acid polymers with the potential to treat or manage a wide range of diseases. Although the majority of oligonucleotide therapeutics have focused on gene silencing, other strategies are being pursued, including splice modulation and gene activation, expanding the range of possible targets beyond what is generally accessible to conventional pharmaceutical modalities. The majority of oligonucleotide modalities interact with their cognate target molecules via complementary Watson-Crick base pairing, and so interrogation of the putative target sequence is relatively straightforward. Highly specific lead compounds can often be rationally designed based on knowledge of the primary sequence of a target gene alone and lead candidates identified by rapid screening. By contrast, conventional small-molecule pharmaceuticals require much larger, and often iterative, screening efforts followed by extensive medicinal chemistry optimization. In addition, the use of oligonucleotides allows for precision and/or personalized medicine approaches as they can theoretically be designed to selectively target any gene with minimal, or at least predictable, off-target effects. Furthermore, it is possible to target patient-specific sequences that are causative of rare disease $^{1}$, specific alleles (for example, SNPs or expanded repeat-containing mutant transcripts can be preferentially targeted without silencing the wild-type $\mathrm{mRNA}^{2-5}$ ), distinct transcript isoforms ${ }^{6}$, pathogenic fusion transcripts (for example, $\mathrm{Bcr}-\mathrm{Abl}^{7}$ ), traditionally 'undruggable' targets (for example, proteins that may lack hydrophobic pockets that may accommodate a small molecule that also inhibits protein activity) ${ }^{8,9}$ and viral sequences that evolve resistance to an oligonucleotide therapy (whereby the oligonucleotide design is modified to compensate for acquired escape mutations) ${ }^{10}$.
In addition to their ability to recognize specific target sequences via complementary base pairing, nucleic acids can also interact with proteins through the formation of three-dimensional secondary structures - a property that is also being exploited therapeutically. For example, nucleic acid aptamers are structured nucleic acid ligands that can act as antagonists or agonists for specific proteins ${ }^{11}$ (BOX 1). Similarly, guide RNA molecules contain hairpin structures that bind to exogenously introduced Cas9 protein and direct it to specific genomic DNA loci for targeted gene editing ${ }^{12}$ (BOX 2). An in-depth discussion of these modalities is beyond the scope of this Review.

As of January 2020, ten oligonucleotide drugs have received regulatory approval from the FDA (FIG. 1; TABLE 1). However, a major obstacle preventing widespread usage of oligonucleotide therapeutics is the difficultly in achieving efficient delivery to target organs and tissues other than the liver. In addition, off-target interactions $^{13-17}$, sequence and chemistry-dependent toxicity and saturation of endogenous RNA processing pathways ${ }^{18}$ must also be carefully considered. The most commonly used strategies employed to improve nucleic acid drug delivery include chemical modification to improve 'drug-likeness', covalent conjugation to cell-targeting or cell-penetrating moieties and nanoparticle formulation. More recently developed approaches such as endogenous vesicle (that is, exosome) loading, spherical nucleic acids (SNAs), nanotechnology applications (for example, DNA cages) and 'smart' materials are also being pursued.

This Review will provide an overview of oligonucleotide-based drug platforms and focus on recent advances in improving oligonucleotide drug delivery. 


\section{Box 1 | Aptamers - evolved nucleic acid ligands}

Aptamers are structured, single-stranded nucleic acid molecules (typically $20-100$ nucleotides) that fold into defined secondary structures and act as ligands that interact with target proteins by way of their three-dimensional structure and adaptive $\mathrm{fit}^{11}$. In contrast with other kinds of nucleic acid therapeutics, aptamers are not rationally designed. Instead, they are generated by an in vitro evolution methodology called SELEX (systematic evolution of ligands by exponential enrichment) ${ }^{286-288}$. Pegaptanib (originally developed by NeXstar Pharmaceuticals and Eyetech Pharmaceuticals) (FIG. 1i; TABLE 1), an RNA-based aptamer that targets the VEGF-165 vascular endothelial growth factor isoform as an anti-angiogenic therapy for neovascular age-related macular degeneration, is currently the only aptamer approved for clinical use.

Aptamers have primarily been used to target extracellular targets (for example, receptors), which somewhat simplifies the delivery problem for this class of oligonucleotide. However, as with other RNA species, RNA aptamers are rapidly degraded in most extracellular environments, meaning that chemical modification of aptamers is essential for in vivo activity. SELEX can be performed with libraries of chemically modified RNAs to a limited extent, as some nucleotide analogues, such as 2'-fluoro and 2'-O-methyl, are tolerated in both reverse transcriptase and T7 RNA polymerase enzymatic steps ${ }^{289,290}$. The introduction of post-SELEX chemical modifications is an alternative approach to further enhance aptamer drug-like properties.

The inherent chirality of amino acids in nature, in turn, enforces chirality in enzymatically produced nucleic acids (that is, L-amino acids and D-nucleotides). However, SELEX can be performed using enantiomeric protein analogues of target proteins synthesized with unnatural $D$-amino acids. The resulting aptamers are necessarily composed of D-RNA as a consequence of the restrictions of the enzymatic SELEX steps. However, the L-RNA versions of these identified aptamers can now be generated by chemical synthesis, which will thereby recognize the natural L-protein. These highly stable 'mirror image' aptamers are called spiegelmers (or L-RNA aptamers) and are not substrates for natural nucleases ${ }^{291}$.

\section{Spliceosome \\ A large riboprotein complex that mediates the splicing of mRNA transcripts}

Nonsense-mediated decay A cellular pathway through which mRNA transcripts containing premature termination codons are eliminated.

\section{RNAi}

A cellular pathway through which small interfering RNAs mediate gene silencing via the slicing of target mRNA transcripts. Much of the RNA machinery is shared with the miRNA processing pathway.

\section{Oligonucleotide-based platforms}

Antisense oligonucleotides. Antisense oligonucleotides (ASOs) are small ( 18-30 nucleotides), synthetic, single-stranded nucleic acid polymers of diverse chemistries, which can be employed to modulate gene expression via various mechanisms. ASOs can be subdivided into two major categories: RNase $\mathrm{H}$ competent and steric block. The endogenous RNase H enzyme RNASEH1 recognizes RNA-DNA heteroduplex substrates that are formed when DNA-based oligonucleotides bind to their cognate mRNA transcripts and catalyses the degradation of RNA ${ }^{19}$. Cleavage at the site of ASO binding results in destruction of the target RNA, thereby silencing target gene expression (FIC. 2a). This approach has been widely used as a means of downregulating disease-causing or disease-modifying genes ${ }^{20}$. To date, three RNase $\mathrm{H}$-competent ASOs have received regulatory approval; fomivirsen, mipomersen and inotersen (FIG. $1 \mathrm{a}-\mathrm{C}$; TABLE 1).

Current-generation RNase H-competent ASOs generally follow the 'gapmer' pattern, whereby a central DNA-based 'gap' is surrounded by RNA-based (but chemically modified) flanking regions that promote target binding ${ }^{21}$ (FIG. 1b,c). Notably, RNASEH1 is active in both the cytoplasm and the nucleus ${ }^{22,23}$, thereby enabling the targeting of nuclear transcripts (for example, immature pre-mRNAs and long non-coding RNAs) that may be less accessible to other technologies (for example, small interfering RNA (siRNA); see below).

Steric block oligonucleotides are ASOs that are designed to bind to target transcripts with high affinity but do not induce target transcript degradation as they lack RNase H competence. Such oligonucleotides therefore comprise either nucleotides that do not form RNase $\mathrm{H}$ substrates when paired with RNA or a mixture of nucleotide chemistries (that is, 'mixmers') such that runs of consecutive DNA-like bases are avoided.

Steric block oligonucleotides can mask specific sequences within a target transcript and thereby interfere with transcript RNA-RNA and/or RNA-protein interactions. The most widely used application of steric block ASOs is in the modulation of alternative splicing in order to selectively exclude or retain a specific exon(s) (that is, exon skipping and exon inclusion, respectively). In these cases, the oligonucleotide 'masks' a splicing signal such that it becomes invisible to the spliceosome, leading to alterations in splicing decisions ${ }^{24,25}$. Typically, such splice correction approaches have been used to restore the translational reading frame in order to rescue production of a therapeutic protein ${ }^{26,27}$. However, the same technology can also be used for splice corruption, whereby an exon is skipped in order to disrupt the translation of the target gene ${ }^{28}$ (FIG. 2b). Given that alternative splicing is responsible for much proteomic diversity, it is possible that steric block oligonucleotides may also be utilized to promote isoform switching, thereby diminishing the expression of harmful protein isoforms and/or promoting the expression of beneficial ones. To date, three splice-switching ASOs have been FDA-approved; eteplirsen, golodirsen and nusinersen (FIG. 1d-f).

Notably, steric block ASOs have also been demonstrated to inhibit translation inhibition ${ }^{29,30}$ (FIG. 2C), interfere with upstream open reading frames that negatively regulate translation ${ }^{31}$ in order to activate protein expression $^{32}$ (FIG. 2d), inhibit nonsense-mediated decay in a gene-specific manner by preventing assembly of exon junction complexes ${ }^{33}$ and influence polyadenylation signals to increase transcript stability ${ }^{34}$ (FIG. 2e).

RNAi-precision duplex silencers. siRNA molecules are the effector molecules of RNAi and classically consist of a characteristic $19+2 \mathrm{mer}$ structure (that is, a duplex of two 21-nucleotide RNA molecules with 19 complementary bases and terminal 2-nucleotide $3^{\prime}$ overhangs) ${ }^{35}$. One of the strands of the siRNA (the guide or antisense strand) is complementary to a target transcript, whereas the other strand is designated the passenger or sense strand. siRNAs act to guide the Argonaute 2 protein (AGO2), as part of the RNA-induced silencing complex (RISC), to complementary target transcripts. Complete complementarity between the siRNA and the target transcript results in cleavage (that is, slicer activity) of the target opposite position 10-11 of the guide strand, catalysed by AGO2 $\left(\mathrm{REFS}^{36-38}\right)$, leading to gene silencing (FIG. 2f). As of May 2020, two siRNAs have received FDA approval: patisiran and givosiran (FIG. $1 \mathrm{~g}, \mathrm{~h}$; TABLE 1).

Numerous variations of the archetypal siRNA design have provided benefits in terms of reduced passenger strand activity and/or improved potency. These include Dicer substrate siRNAs $s^{39}$, small internally segmented siRNAs ${ }^{40}$, self-delivering siRNAs (asymmetric and hydrophobic $)^{41}$, single-stranded siRNAs ${ }^{42,43}$ and divalent siRNAs ${ }^{44}$. 
microRNA inhibition. microRNAs (miRNAs) are endogenous RNAi triggers that have been implicated in a multitude of physiological and pathophysiological processes, including cancer ${ }^{45,46}$, cell cycle progression ${ }^{47}$, infectious disease ${ }^{48,49}$, immunity ${ }^{50}$, diabetes ${ }^{51}$, metabolism ${ }^{52}$, myogenesis ${ }^{53,54}$ and muscular dystrophy ${ }^{55,56}$. miRNAs therefore constitute a rich class of putative drug targets. miRNA hairpins embedded within long primary miRNA transcripts are sequentially processed by two RNase III family enzymes, DICER1 (Dicer) and DROSHA, which liberate the hairpin and then cleave the loop sequence, respectively ${ }^{37,57,58}$. The resulting duplex RNA (analogous to an siRNA) is loaded into an argonaute protein (for example, AGO2) and one strand discarded to generate the mature, single-stranded miRNA species. As with siRNAs, miRNAs guide RISC to target sequences where they initiate gene silencing. In contrast with siRNAs, miRNAs typically bind with partial complementarity and induce silencing via slicer-independent mechanisms ${ }^{59,60}$.

Steric block ASOs have been extensively utilized to competitively inhibit miRNAs via direct binding to the small RNA species within the RISC complex ${ }^{61}$ (FIG. 2g). Such ASOs are known as anti-miRNA oligonucleotides, anti-miRs or antagomirs ${ }^{62}$. The first anti-miRNA drug to enter clinical trials was miravirsen ${ }^{63}$ (later called SPC3649, Santaris Pharma A/S/Roche; TABLE 2), which is an ASO designed to treat chronic hepatitis $\mathrm{C}$ virus (HCV) infection via targeting of the liver-specific miRNA miR-122. This miRNA binds to two sites in the 5 ' untranslated region of the HCV viral RNA and thereby stabilizes it ${ }^{48,64-68}$. Miravirsen sequesters miR-122, leaving the viral RNA exposed to exonucleolytic degradation with a concomitant failure of the virus to replicate

\section{Box 2 | Nucleic acid-programmable nucleases}

The discovery that the CRISPR-Cas9 system could be repurposed for use in mammalian cells has led to a renaissance in the field of gene editing ${ }^{12}$. This system, which evolved as a form of bacterial immune defence against invading bacteriophage ${ }^{292-294}$, consists of a protein component (that is, the Cas9 enzyme that introduces double-stranded DNA breaks) and one or more guide RNA components (for example, the single guide RNA that directs Cas 9 to a specific target site in genomic DNA). The modularity of the system allows for testing of many potential single guide RNAs, whereas the protein component is invariant. By contrast, previous gene editing approaches (for example, meganuclease, zinc finger nucleases and TALENs (transcription activator-like effector nucleases)) required costly and time-consuming protein engineering in order to confer target specificity. The CRISPR-Cas9 system induces double-strand breaks at specific genomic DNA loci, which are subsequently repaired by one of several DNA repair mechanisms. Such editing can be used to knockout a gene by disrupting the translational reading frame, excise a specific region from the genome, repair a point mutation or knock-in a desired DNA sequence. Furthermore, nuclease-deficient Cas 9 variants ( $\mathrm{dCas} 9$ ) have been developed that interact with target DNA sequences but do not induce double-strand breaks. dCas 9 fusions with transcriptional activation (VPR; VP64-p65-Rta) ${ }^{295}$ or silencing proteins (KRAB) ${ }^{296}$ can therefore be used to target these fusion proteins to specific promoter sequences, and thereby modulate gene expression without inducing permanent changes in the DNA sequence. The majority of CRISPRCas9 gene editing therapies are dependent on the use of viral vectors for delivery of the effector components. The development of a compact Cas 9 enzyme derived from Staphylococcus aureus ( $\mathrm{SaCas} 9$ ) has enabled the delivery of the CRISPR system using adeno-associated viral vectors ${ }^{297}$. However, non-viral approaches using Cas9 ribonucleoprotein complexes loaded with synthetic oligonucleotide guide RNAs are also being developed ${ }^{298}$. These include gold nanoparticles (CRISPR-gold) ${ }^{299}$, liposomes ${ }^{300,301}$ and cell-penetrating peptide-modified Cas $9\left(\mathrm{REF}^{302}\right)$. and a reduction in viral load. Despite miravirsen showing promising results in terms of viral suppression, a rebound in HCV load was eventually observed in patient serum ${ }^{67}$, and an escape mutation that renders the HCV genome refractory to miravirsen has also been reported $^{69}$. A rival anti-miR-122 drug, RG-101 (Regulus Therapeutics), for the treatment of HCV infection was similarly developed, but both miravirsen/SPC3649 and RG-101 are no longer in clinical development. Notably, Regulus is also developing anti-miRNA oligonucleotide drugs targeting miR-21 for Alport nephropathy ${ }^{70}$ and miR-17 for polycystic kidney disease ${ }^{71}$ (TABLE 2). Similarly, miRagen Therapeutics is developing cobomarsen, an oligonucleotide inhibitor of miR-155 for the treatment of cutaneous $\mathrm{T}$ cell lymphoma ${ }^{72}$, and remlarsen, a double-stranded mimic of miR-29 for the treatment of keloids ${ }^{73}$ (TABLE 2).

An alternative approach to miRNA inhibition is the use of steric block ASOs to inhibit a specific miRNA regulatory interaction via masking of the target sequence on an mRNA transcript ${ }^{74,75}$ (FIG. $1 \mathrm{~g}$ ). However, the potential of steric block ASOs to disrupt these and other trans-acting regulators of RNA expression has yet to be fully realized.

Transcriptional gene activation. Many gene loci express long non-coding RNA species (for example, promoter-associated RNA and natural antisense transcripts, NATs) that are often involved in the transcriptional repression of the proximal protein-coding gene (or genes) ${ }^{76}$. Targeting these long non-coding RNAs with ASOs or siRNAs (referred to as antagoNATs ${ }^{77}$ or small activating $\mathrm{RNAs}^{78}$ ) can reverse the effects of this negative regulation leading to transcriptional activation (or 'unsilencing'; FIG. 2h), as has been shown in the case of numerous disease-relevant genes including BACE1 (Alzheimer disease) $^{79}, B D N F$ (Parkinson disease) ${ }^{80}$, UBE3A (Angelman syndrome) ${ }^{81}$ and SCN1A (Dravet syndrome $)^{82}$, among others. Alternatively, small activating RNAs can recruit epigenetic remodelling complexes to activate transcription via a distinct mechanism ${ }^{83,84}$ (FIG. 2i). Similarly, there is a growing appreciation of the importance of endogenous small RNAs in the nucleus that function as natural mediators of such transcriptional gene activation or silencing events, and may themselves constitute targets for oligonucleotide therapeutics ${ }^{85,86}$.

MiNA Therapeutics is currently developing MTLCEBPA, a small activating RNA targeting CEBPA ${ }^{87,88}$ (CCAAT/enhancer-binding protein- $\alpha$, a key transcription factor involved in hepatocyte differentiation and tumour suppression) delivered as a lipid nanoparticle formulation, as a treatment for hepatocellular carcinoma (TABLE 2). This drug is the first gene-activating oligonucleotide to be tested in a phase I clinical trial in patients with hepatocellular carcinoma and cirrhosis ${ }^{89}$.

An alternative approach for specific gene activation is the TANGO (Targeted Augmentation of Nuclear Gene Output) method currently under development by Stoke Therapeutics (TABLE 2). This strategy takes advantage of naturally occurring non-productive alternative splicing events, which result in premature termination 
a Fomivirsen

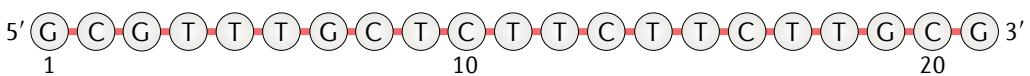

b Mipomersen

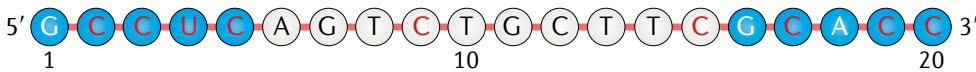

c Inotersen

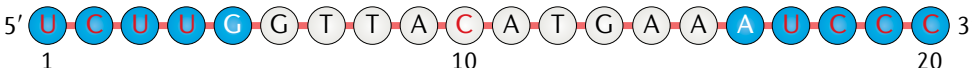

d Eteplirsen

5' C-T-C-C-A-A-C-A-T-C-A-A-G-C-A-A-C-A-T-G-C-C-A-T-T-T-C-T-A-C $3_{10}^{\prime \prime}$

e Golodirsen

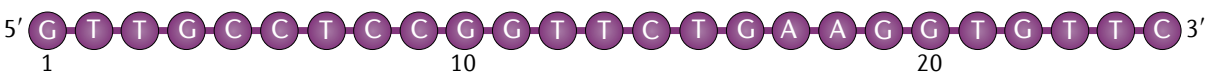

f Nusinersen

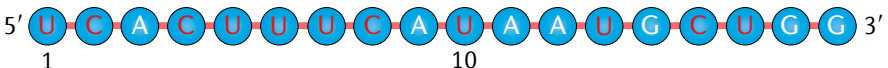

g Patisiran

Passenger strand

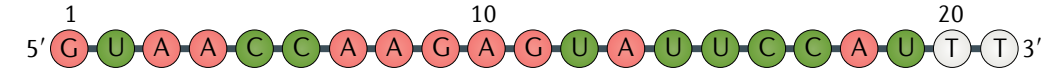

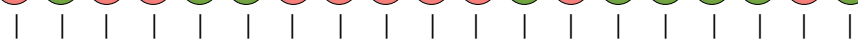

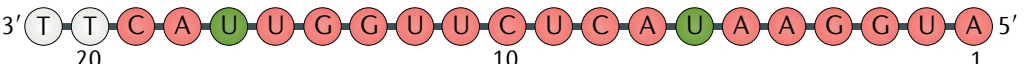

Guide strand

h Givosiran

Passenger strand
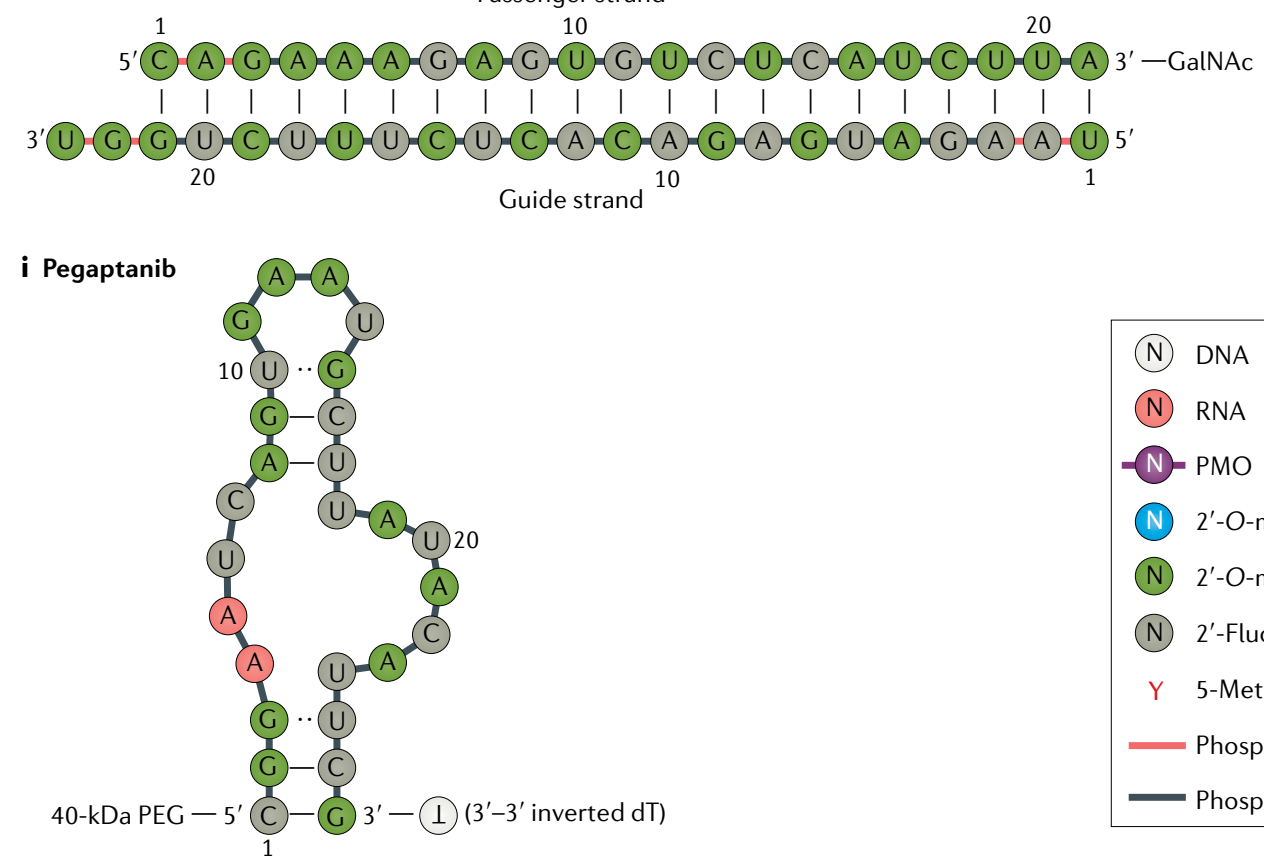

\begin{tabular}{|ll|}
\hline (N) DNA \\
(N) RNA \\
(N) PMO \\
(N) 2'-O-methoxyethyl \\
(N) 2'-O-methyl \\
(N) 2'-Fluoro \\
Y 5-Methyl pyrimidine \\
C Phosphorothioate \\
C Phosphodiester
\end{tabular}

Fig. 1 | Chemistry of FDA-approved oligonucleotide drugs. Chemical composition of the FDA-approved oligonucleotide drugs fomivirsen (part a), mipomersen (part b), inotersen (part c), eteplirsen (part d), golodirsen (part e), nusinersen (part f), patisiran (part g), givosiran (part h) and pegaptanib (part i). Drugs are ordered by mechanism of action. Drug names, trade names, principal developing company, modality and RNA target are described in TABLE 1 for each compound. The drug defibrotide consists of a mixture of single-stranded and double-stranded ribonucleotides of variable length and sequence composition harvested from pig intestine. It therefore cannot be easily represented in the same manner as the other oligonucleotide drugs and so is not shown here. GalNAc, $\mathrm{N}$-acetylgalactosamine; PEG, polyethylene glycol; PMO, phosphorodiamidate morpholino oligonucleotide. Part i structure adapted from REF. ${ }^{284}$, Springer Nature Limited. 
Table 1 | FDA-approved oligonucleotide therapeutics

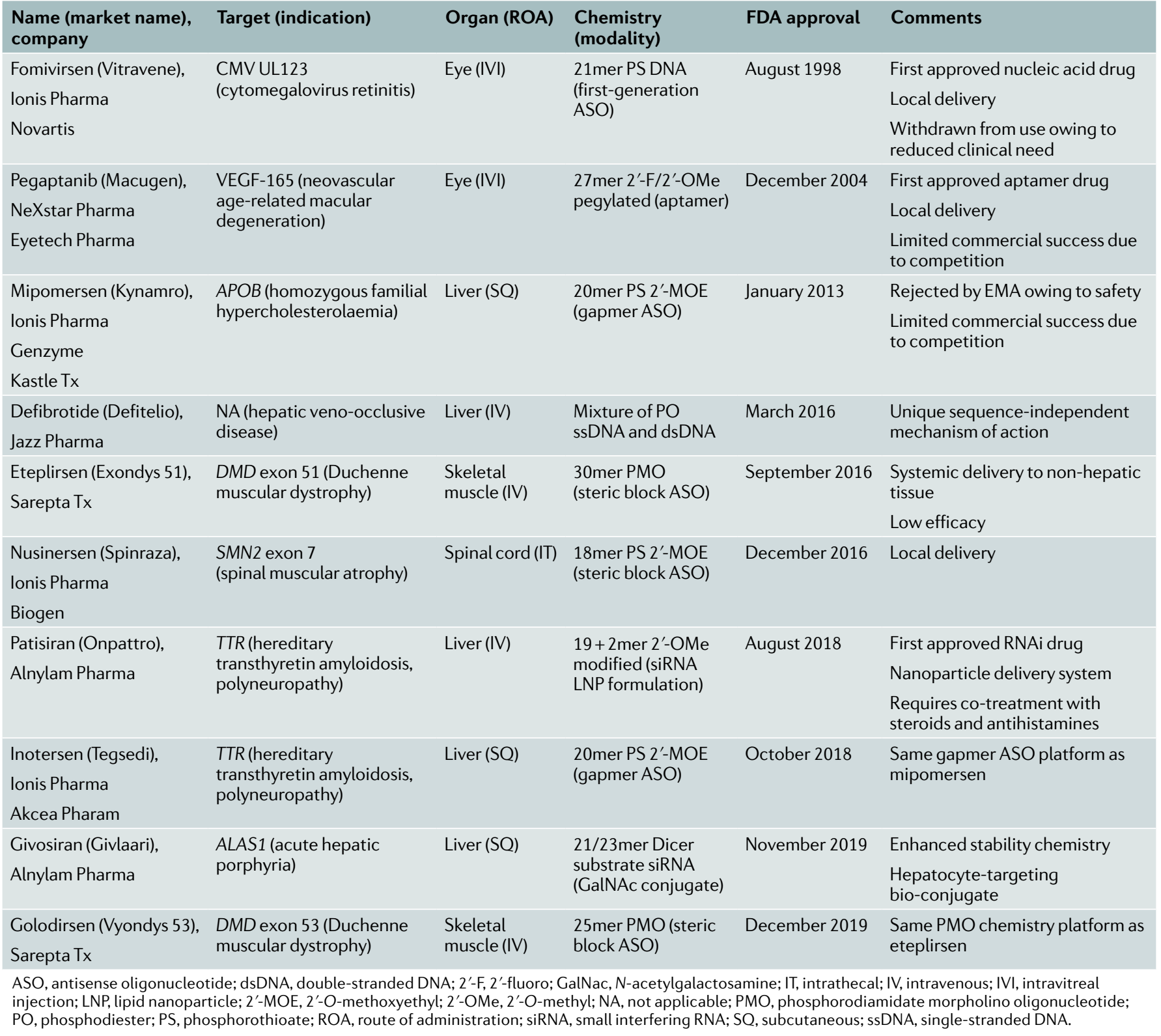

codon generation and transcript degradation via nonsense-mediated decay. Splice-correcting ASOs are targeted to the sites of non-productive alternative splicing products to promote the generation of the productive transcript isoform, thereby upregulating target gene expression (Stoke Therapeutics' science).

\section{Exocytosis}

A cellular mechanism in which molecules are exported from the cell in an energy-dependent manner. This is achieved through the fusion of intracellular vesicles with the plasma membrane, thereby secreting their contents into the extracellular space. Vesicles released in this manner are called exosomes.

\section{Delivery challenges}

Achieving effective delivery of oligonucleotide therapeutics to many tissues remains a major translational challenge. Oligonucleotides are typically large, hydrophilic polyanions (single-stranded ASOs are $\sim 4-10 \mathrm{kDa}$, double-stranded siRNAs are $\sim 14 \mathrm{kDa}$ ), properties that mean they do not readily pass through the plasma membrane. For activity, systemically injected nucleic acid drugs must resist nuclease degradation in the extracellular space ${ }^{90}$, bypass renal clearance ${ }^{91,92}$, evade non-productive sequestration by certain plasma proteins $\mathrm{s}^{93}$, avoid removal by the reticuloendothelial system (that is, mononuclear phagocytes, liver sinusoidal endothelial cells and Kupffer cells) ${ }^{94}$, cross the capillary endothelium at the desired target cell(s) within an organ/tissue by paracellular or transcellular routes, traverse the plasma membrane, escape the endolysosomal system before lysosomal degradation or re-export via exocytosis ${ }^{95}$ and arrive at the correct intracellular site of action. Systemic delivery to the central nervous system (CNS) presents an additional obstacle, as oligonucleotide-based therapeutics are generally not able to traverse the blood-brain barrier (BBB).

To date, the majority of oligonucleotide therapeutics (and almost all of the approved nucleic acid drugs) have focused on either local delivery (for example, to the eye or spinal cord) or delivery to the liver. The eye is considered an immune-privileged organ, meaning that this has been an anatomical target of choice for gene and 


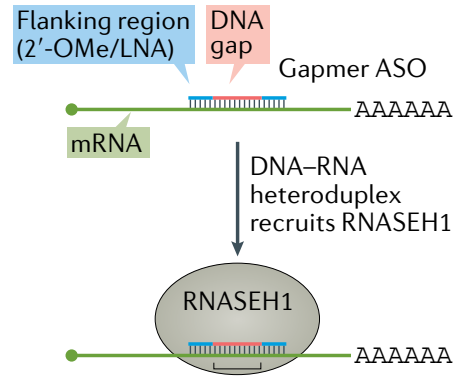

Transcript cleavage and degradation b

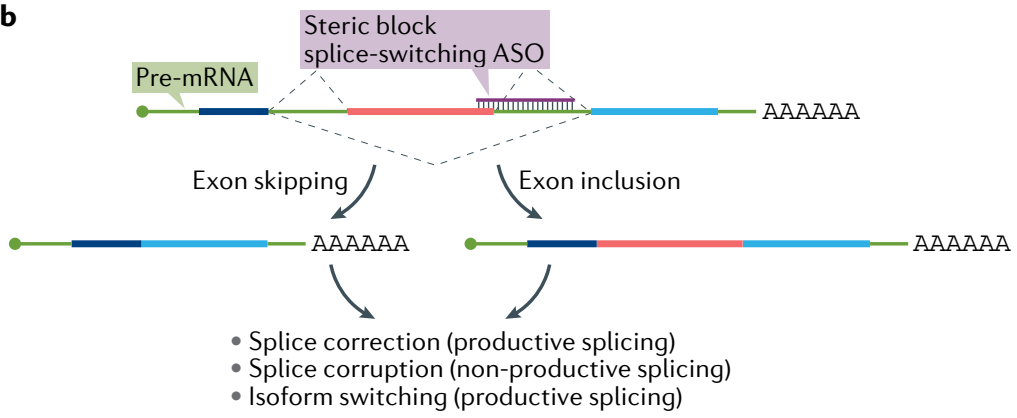

C Ribosome<smiles>Cc1ccccc1</smiles>

\section{Steric block ASO}

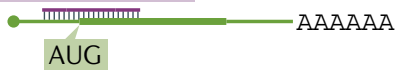

d uORF peptide induces translational repression of $\mathrm{pORF}$

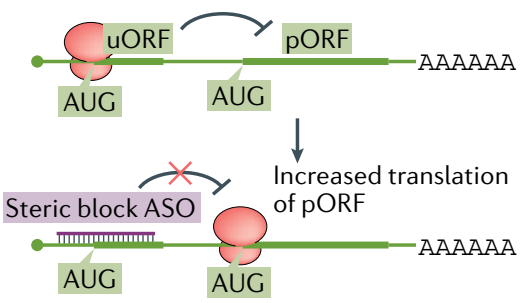

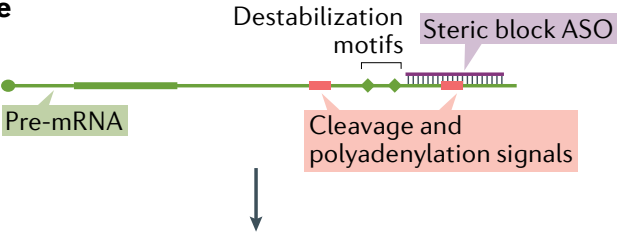

AAAAAA

Early addition of polyadenyl tail results in more stable transcript lacking destabilization signals f

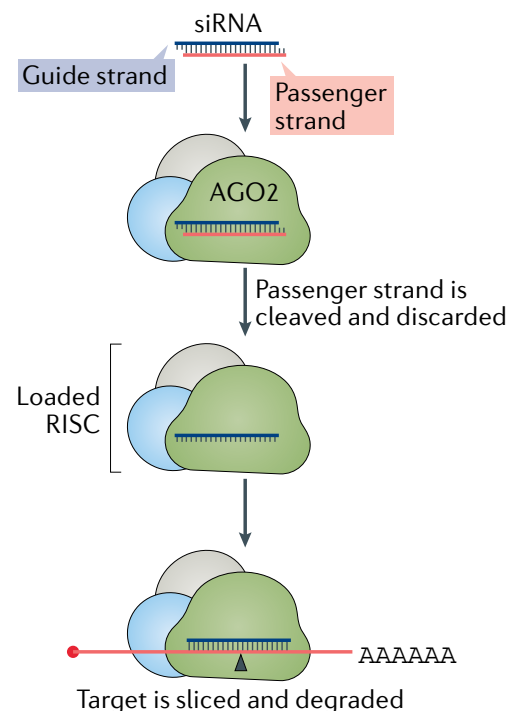

h
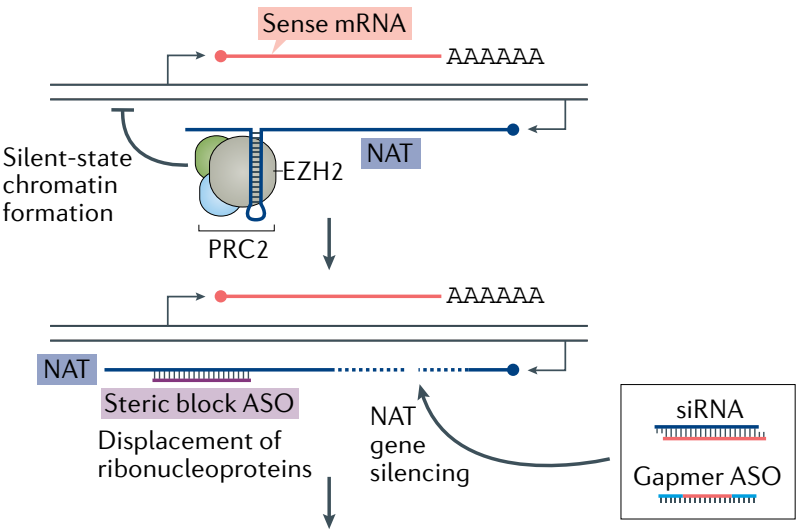

Transcriptional activation of sense gene

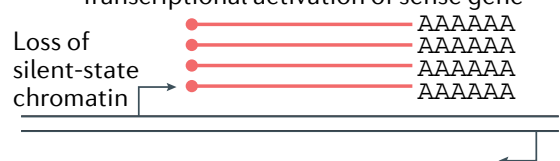

g Endogenous pre-miRNA

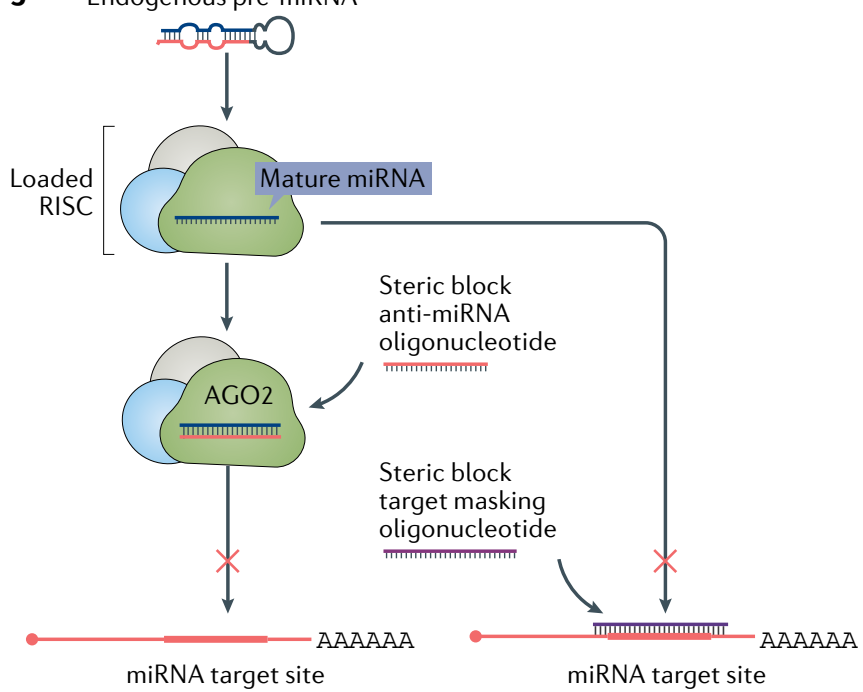

i

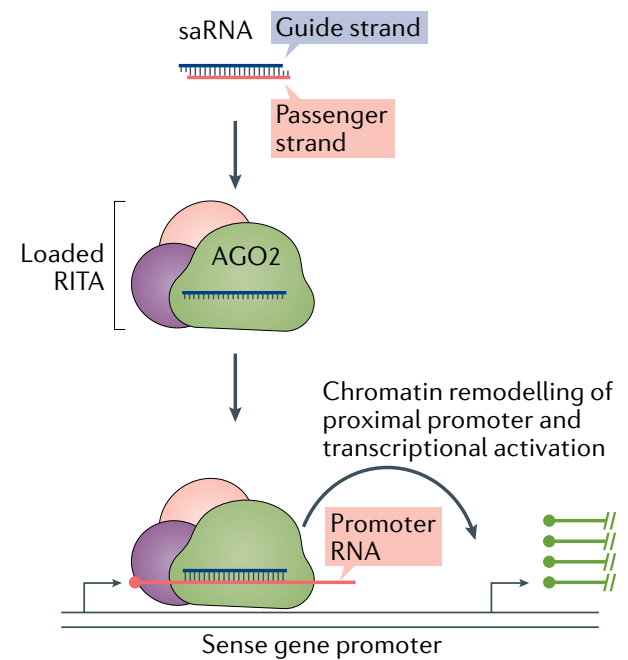


4 Fig. 2 | Oligonucleotide-mediated gene regulatory mechanisms. a | Gapmer antisense oligonucleotides (ASOs), consisting of a DNA-based internal 'gap' and RNA-like flanking regions (often consisting of 2'-O-methyl (2'-OMe) or locked nucleic acid (LNA) modified bases) bind to target transcripts with high affinity. The resulting RNA-DNA duplex acts as a substrate for RNASEH1, leading to the degradation of the target transcript. $\mathbf{b}$ | Steric block oligonucleotides targeted to pre-mRNA splicing signals modulate alternative splicing to either promote exon skipping or exon inclusion (depending on the type of splicing signal targeted). The resulting mature mRNA species can be spliced in a productive manner (for example, to restore the reading frame or to switch to an alternative isoform) or in a non-productive manner (for example, to remove an exon that is required for protein function and/or to disrupt the translation reading frame). $\mathbf{c}$ (Steric block antisense oligonucleotides can disrupt translation initiation by targeting the AUG start codon. $\mathbf{d}$ | Some transcripts contain upstream open reading frames (uORFs) that modulate the translational activity of the primary open reading frame (pORF). Targeting the uORF with steric block ASOs disrupts this regulation, leading to activation of pORF translation. e | Transcript stability can be modulated by shifting the usage of cleavage and polyadenylation signals. For example, a steric block ASO targeted to a distal polyadenylation signal results in the preferential usage of a weaker proximal polyadenylation signal. The resulting shorter transcript is more stable as it lacks RNA destabilization signals. $\mathbf{f} \mid$ Small interfering RNAs (siRNAs) enter the RNA-induced silencing complex (RISC), which consists of Argonaute 2 protein (AGO2), DICER1 and TARBP2, and the passenger strand is discarded. The guide strand directs the RISC to complementary target genes that are cleaved by the slicer activity of AGO2. g| Endogenous microRNAs (miRNAs) are loaded into miRISC. miRNA activity can be inhibited by steric block ASOs that either complex with the mature miRNA loaded in the RISC complex or by masking a target site through interactions with the targeted transcript. $\mathbf{h}$ | Natural antisense transcripts (NATs) recruit epigenetic silencing complexes, such as PRC2, to a sense gene locus. Interference of the epigenetic modifier protein association with the NAT using steric block ASOs or degradation of the NAT via siRNA or gapmer ASO results in 'unsilencing' of the sense gene. $\mathbf{i}$ | Small activating RNAs (saRNAs) can recruit the RNA-induced transcriptional activation (RITA) complex (consisting of AGO2, CTR9 and DDX5 (REF. ${ }^{285}$ )) to low-copy promoter-associated RNA, leading to transcriptional activation of the proximal gene. EZH2, Enhancer of zeste homolog 2; PRC2, polycomb repressive complex 2.

Blood-brain barrier

(BBB). A physical barrier that selectively prevents molecules and pathogens from crossing from the blood and into the extracellular space in the brain and spinal cord. The bloodbrain barrier is composed of blood capillary endothelial cells, pericytes and astrocyte end-feet. oligonucleotide therapies (for example, pegaptanib and fomivirsen). Conversely, direct injection of oligonucleotides into the cerebrospinal fluid via lumbar puncture has been demonstrated to result in a favourable distribution of therapeutic molecules throughout the CNS (for example, nusinersen) ${ }^{96}$. The liver is a highly perfused tissue, with a discontinuous sinusoidal endothelium, meaning that uptake of free oligonucleotides and larger nanoparticles can occur rapidly before renal clearance. The liver also contains very high concentrations of receptors that can facilitate uptake and/or are rapidly recycled (for example, scavenger receptors and the asialoglycoprotein receptor). Although other highly vascularized tissues with discontinuous or fenestrated endothelia, such as the kidneys and spleen, are also sites for oligonucleotide accumulation, the development of effective technologies for extrahepatic systemic delivery remains a major goal for the oligonucleotide therapeutics field.

\section{Strategies to enhance delivery}

Chemical modification. Chemical modification represents one of the most effective approaches to enhance oligonucleotide drug delivery. Modification of the nucleic acid backbone, the ribose sugar moiety and the nucleobase itself has been extensively employed in order to improve the drug-like properties of oligonucleotide drugs and thereby enhance delivery 92,97 $^{92}$ (FIG. 3). Specifically, modification is utilized to improve oligonucleotide pharmacokinetics, pharmacodynamics and biodistribution. Specific patterns of modification are also required for the functionality of certain therapeutic modalities (for example, gapmers). The importance of chemistry is exemplified by the observation that extensive chemical modification of second-generation gapmer ASOs is sufficient to enable delivery to a wide variety of tissues, without the need for an additional delivery agent ${ }^{98}$. Furthermore, of the ten approved oligonucleotide therapies approved to date (TABLE 1), eight are 'naked' (that is, lacking an additional delivery vehicle) and so are dependent on chemical modification alone to facilitate their tissue delivery. This is also true of gapmer ASOs currently in development by Ionis Pharmaceuticals, including drugs for the treatment of amyotrophic lateral sclerosis, Alzheimer disease, centronuclear myopathy and, most notably, Huntington disease $^{99}$ (TABLE 2).

Backbone modification. The incorporation of phosphorothioate (PS) linkages (FIG. 3), in which one of the non-bridging oxygen atoms of the inter-nucleotide phosphate group is replaced with sulfur, is widely used in therapeutic oligonucleotides ${ }^{100}$. There are many other kinds of backbone modification (for example, boranophosphate ${ }^{101}$ ), although these have been less commonly used. PS backbone modifications are readily tolerated in ASO designs and do not disrupt RNase $\mathrm{H}$ activity. By contrast, siRNAs that contain PS modifications at every linkage are less active than the equivalent phosphodiester (PO) siRNA ${ }^{102}$, and, as such, PS-containing siRNAs are typically modified at the termini only, if at all. Sulfated molecules, such as oligonucleotides containing PS linkages or thiol tails, are also taken up by scavenger receptors (such as the stabilins STAB1 and STAB2), which mediate their internalization into tissues such as the liver ${ }^{103-105}$. The incorporation of PS linkages has the dual effect of conferring nuclease resistance and promoting binding to proteins in both plasma and within cells. Oligonucleotide interactions with plasma proteins such as albumin ${ }^{106}$ have the effect of improving drug pharmacokinetics by increasing the circulation time (and therefore reducing renal clearance). However, binding of a PS-containing gapmer ASO to plasma a2-macroglobulin (A2M) was found to be non-productive ${ }^{93}$. PS modification of oligonucleotides also increases interactions with intracellular proteins (for example, nucleolin ${ }^{107-111}$ ) that are believed to promote their accumulation in the nucleus, the target site of action for splice-switching oligonucleotides.

Notably, resistance to cellular nucleases results in prolonged tissue retention and long-lasting drug effects. In cases where this is undesirable, in the case of toxicity due to prolonged gene silencing for example, the incorporation of one or more PO linkages can be used to 'tune' the durability of the oligonucleotide by reducing its nuclease stability ${ }^{112}$.

A disadvantage of PS backbone modifications is that they have the effect of reducing the binding affinity of an oligonucleotide for its target, a limitation that can be compensated for by incorporating additional types of modification (discussed below). 
Table 2 | Selected oligonucleotide therapeutics that have entered development

\begin{tabular}{|c|c|c|c|c|c|}
\hline Company & Drug (partner) & $\begin{array}{l}\text { Modality/delivery } \\
\text { technology }\end{array}$ & Target/organ & Indication & $\begin{array}{l}\text { Clinical trial } \\
\text { stage }\end{array}$ \\
\hline \multirow[t]{6}{*}{$\begin{array}{l}\text { lonis } \\
\text { Pharmaceuticals }\end{array}$} & $\begin{array}{l}\text { IONIS-HTTRx/RG6042 } \\
\text { (Roche) }\end{array}$ & ASO/none & HTT/brain & Huntington disease & Phase III \\
\hline & Tofersen (Biogen) & ASO/none & $\begin{array}{l}\text { SOD1/brain and } \\
\text { spinal cord }\end{array}$ & ALS & Phase III \\
\hline & IONIS-C9Rx & ASO/none & $\begin{array}{l}\text { C9ORF72/brain and } \\
\text { spinal cord }\end{array}$ & ALS & Phase II \\
\hline & IONIS-MAPTRx & ASO/none & MAPT/brain & Alzheimer disease/FTD & Phase II \\
\hline & $\begin{array}{l}\text { IONIS-DNM2-2.5Rx } \\
\text { (Dynacure) }\end{array}$ & ASO/none & DNM2/muscle & Centronuclear myopathy & Phase I \\
\hline & Undisclosed & ASO/none & $\begin{array}{l}\text { Various targets/heart } \\
\text { and tumours }\end{array}$ & $\begin{array}{l}\text { Various rare diseases, } \\
\text { cardiometabolic disorders } \\
\text { and cancers }\end{array}$ & Phase II \\
\hline \multirow{2}{*}{$\begin{array}{l}\text { Sarepta } \\
\text { Therapeutics }\end{array}$} & Casimersen & $\mathrm{PMO}$ ASO/none & DMD exon $45 /$ muscle & DMD & Phase III \\
\hline & SRP-5051 & $\begin{array}{l}\text { PPMO ASO/peptide } \\
\text { platform }\end{array}$ & DMD exon $51 /$ muscle & DMD & Phase I \\
\hline $\begin{array}{l}\text { Nippon Shinyaku } \\
\text { Pharma }\end{array}$ & Viltolarsen & ASO/none & DMD exon $53 /$ muscle & DMD & $\begin{array}{l}\text { Phase II } \\
\text { (approved in } \\
\text { Japan) }\end{array}$ \\
\hline \multirow[t]{5}{*}{$\begin{array}{l}\text { Alnylam } \\
\text { Pharmaceuticals }\end{array}$} & $\begin{array}{l}\text { Fitusiran/ALN-AT3 } \\
\text { (Sanofi Genzyme) }\end{array}$ & siRNA/GalNAc platform & SERPINC1/liver & Haemophilia A and B & Phase III \\
\hline & Lumasiran/ALN-GO1 & siRNA/GalNAc platform & HAO1/liver & Primary hyperoxaluria type 1 & Phase III \\
\hline & Vutrisiran/ALN-TTRsc02 & siRNA/GalNAc platform & TTR/liver & Hereditary amyloidosis & Phase III \\
\hline & Revusiran/ALN-TTRSC & siRNA/GalNAc platform & TTR/liver & Hereditary amyloidosis & $\begin{array}{l}\text { Phase III } \\
\text { — discontinued }\end{array}$ \\
\hline & $\begin{array}{l}\text { Inclisiran (Medicines } \\
\text { Company and Novartis) }\end{array}$ & siRNA/GalNAc platform & PCSK9/liver & Hypercholesterolaemia & Phase III \\
\hline \multirow[t]{2}{*}{ Wave Life Sciences } & Suvodirsen & ASO/stereopure & DMD exon $51 /$ muscle & DMD & $\begin{array}{l}\text { Phase III } \\
\text { — discontinued }\end{array}$ \\
\hline & $\begin{array}{l}\text { WVE-120101; } \\
\text { WVE-120102 (Takeda) }\end{array}$ & ASO/stereopure & $\begin{array}{l}\text { Mutant } H T T / \text { brain } \\
\text { and spinal cord }\end{array}$ & Huntingdon disease & Phase I \\
\hline $\begin{array}{l}\text { Quark } \\
\text { Pharmaceuticals }\end{array}$ & QPI-1002 & siRNA/none & TP53/kidney & $\begin{array}{l}\text { Kidney delayed graft } \\
\text { function/acute kidney injury }\end{array}$ & Phase III \\
\hline Sylentis & Tivanisiran & siRNA/none & TRPV1/eye & Dry eye syndrome & Phase III \\
\hline Moderna & AZD8601 (AstraZeneca) & mRNA/none & VEGFA/heart & Cardiac regeneration & Phase II \\
\hline Santaris/Roche & Miravirsen & Anti-miRNA/none & miR-122/liver & Hepatitis $C$ infection & $\begin{array}{l}\text { Phase II } \\
\text { — discontinued }\end{array}$ \\
\hline \multirow[t]{3}{*}{$\begin{array}{l}\text { Regulus } \\
\text { Therapeutics }\end{array}$} & $\begin{array}{l}\text { RG-012 (Sanofi } \\
\text { Genzyme) }\end{array}$ & Anti-miRNA/none & miR-21/kidney & Alport syndrome & Phase II \\
\hline & RGLS4326 & Anti-miRNA/none & miR-17/kidney & $\begin{array}{l}\text { Autosomal dominant } \\
\text { polycystic kidney disease }\end{array}$ & Phase I \\
\hline & RG-101 & $\begin{array}{l}\text { Anti-miRNA/GalNAc } \\
\text { platform }\end{array}$ & miR-122/liver & Hepatitis $C$ infection & $\begin{array}{l}\text { Phase II } \\
\text { — discontinued }\end{array}$ \\
\hline \multirow[t]{2}{*}{ Mirage Therapeutics } & Cobomarsen/MRG-106 & Anti-miRNA/none & miR-155/lymphomas & Cutaneous T cell lymphoma & Phase II \\
\hline & Remlarsen/MRG-201 & miRNA mimic/none & miR-29/skin & Cutaneous fibrosis & Phase II \\
\hline Arbutus Biopharma & AB-729 & $\begin{array}{l}\text { Anti-miRNA/GalNAc } \\
\text { platform }\end{array}$ & $\begin{array}{l}\text { Hepatitis B virus } \\
\text { HBsAg/liver }\end{array}$ & Hepatitis B infection & Phase I \\
\hline $\begin{array}{l}\text { Arrowhead } \\
\text { Pharmaceuticals }\end{array}$ & ARO-AAT & $\begin{array}{l}\text { siRNA/TRiM platform } \\
\text { - GalNAc-related }\end{array}$ & AAT/liver & a1-Antitrypsin deficiency & Phase II \\
\hline Silence Therapeutics & SLN124 & siRNA/GalNAc platform & TMPRSS6/liver & $\beta$-Thalassaemia & Phase I \\
\hline $\begin{array}{l}\text { Dicerna } \\
\text { Pharmaceuticals }\end{array}$ & DCR-PHXC & $\begin{array}{l}\text { siRNA/GalXC platform } \\
\text { — GalNAc-related }\end{array}$ & LDHA/liver & Primary hyperoxaluria & Phase I \\
\hline MiNA Therapeutics & MTL-CEPBA & saRNA/LNP (SMARTICLES) & CEBPA/liver & Hepatocellular carcinoma & Phase I/II \\
\hline Avidity Biosciences & Undisclosed & $\begin{array}{l}\text { siRNA or ASO/antibody } \\
\text { platform }\end{array}$ & DMPK/muscle & Myotonic dystrophy I & Preclinical \\
\hline PepGen Ltd & Undisclosed & $\begin{array}{l}\text { siRNA or ASO/peptide } \\
\text { platform }\end{array}$ & $\begin{array}{l}\text { Undisclosed target/ } \\
\text { muscle and central } \\
\text { nervous system }\end{array}$ & Neuromuscular disease & Preclinical \\
\hline Stoke Therapeutics & Undisclosed & ASO/none & SCN1A/brain & Dravet syndrome & Preclinical \\
\hline
\end{tabular}

ALS, amyotrophic lateral sclerosis; ASO, antisense oligonucleotide; DMD, Duchenne muscular dystrophy; FTD, frontotemporal dementia; GalNAc,

$\mathrm{N}$-acetylgalactosamine; LNP, lipid nanoparticle; miRNA, microRNA; PMO, phosphorodiamidate morpholino oligonucleotide; PPMO, peptide-PMO; saRNA, small activating RNA; siRNA, small interfering RNA. 

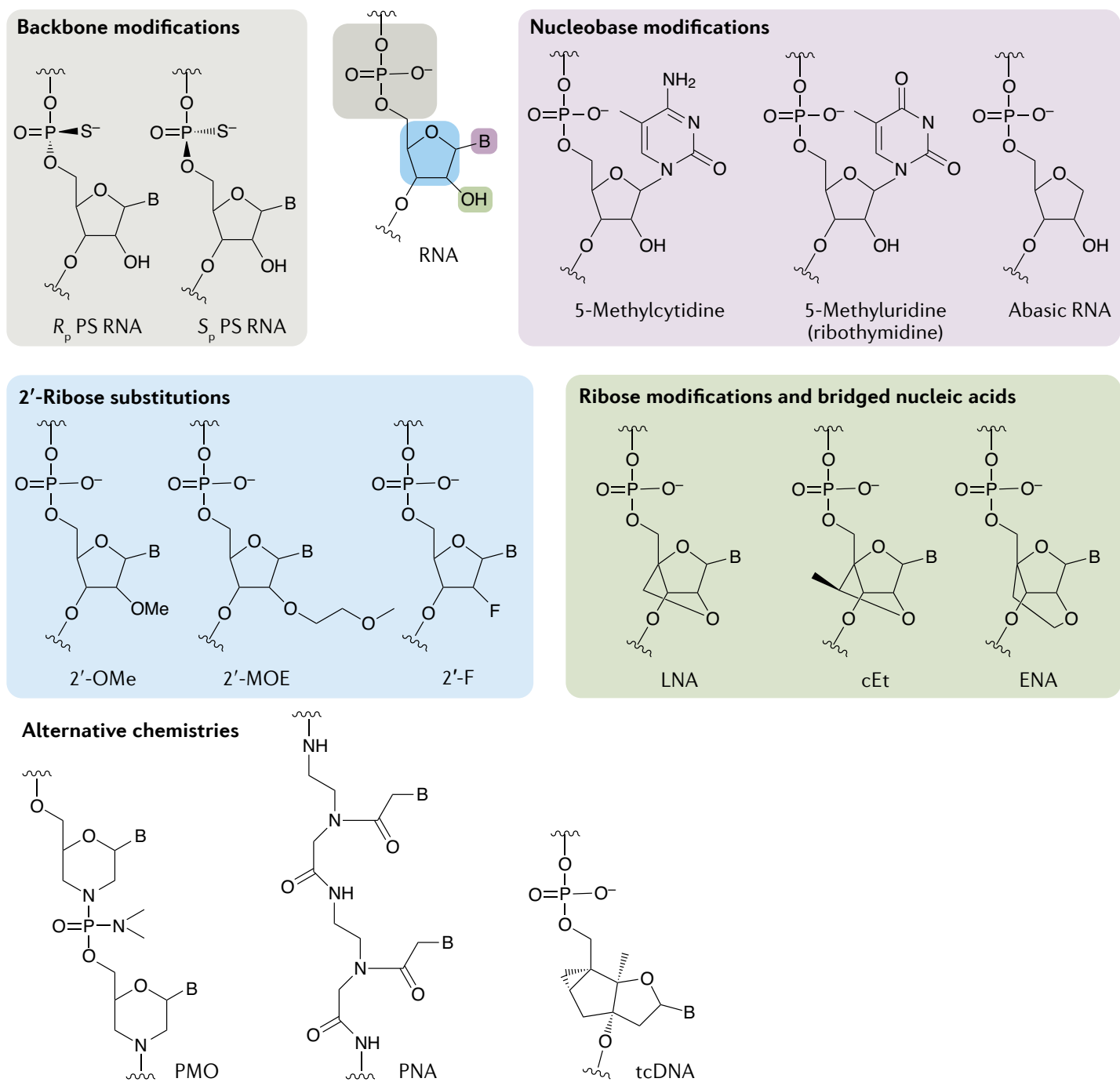

Fig. 3 | Common chemical modifications used in oligonucleotide drugs. Schematic of an RNA nucleotide and how it can be chemically modified at the backbone, nucleobase, ribose sugar and 2 '-ribose substitutions. B, nucleobase; cEt, constrained ethyl bridged nucleic acid; ENA, ethylene-bridged nucleic acid; 2'-F, 2'-fluoro; LNA, locked nucleic acid; 2'-MOE, 2'-O-methoxyethyl; 2'-OMe, 2'-O-methyl; PMO, phosphorodiamidate morpholino oligonucleotide; PNA, peptide nucleic acid; PS, phosphorothioate; tcDNA, tricyclo DNA.

Stereochemistry. The introduction of an additional sulfur atom in a PS linkage results in the generation of a chiral centre at each modified phosphorous atom, with the two possible stereoisomeric forms (designated $S_{\mathrm{p}}$ and $R_{\mathrm{p}}$, respectively) (FIG. 3). As such, a fully PS backbone 20 mer oligonucleotide is in fact a racemic mixture of the $2^{19}$ possible permutations (that is, more than half a million different molecules). The physicochemical properties of each stereocentre are distinct in terms of hydrophobicity/ionic character, nuclease resistance, target affinity and RNase $\mathrm{H}$ activity ${ }^{113}$. In particular, a $3^{\prime}-S_{\mathrm{p}} S_{\mathrm{p}} R_{\mathrm{p}}-5^{\prime}$ 'stereochemical code' contained within the 'gap' region of gapmer ASOs was found to be particularly active $^{113}$. Wave Life Sciences has developed a scalable method of synthesizing oligonucleotides with defined stereochemistry at each PS linkage ${ }^{113}$, and is advancing oligonucleotide drugs with defined stereochemistry for various indications. However, they recently discontinued development of suvodirsen, a stereopure
ASO designed to treat Duchenne muscular dystrophy (DMD) via skipping of dystrophin exon 51, owing to lack of efficacy in a phase I clinical trial ${ }^{114}$. Parallel clinical programmes with stereopure oligonucleotides targeting Huntington disease and C9ORF72 amyotrophic lateral sclerosis/frontotemporal dementia are ongoing (TABLE 2).

It is intriguing to speculate that the racemic mixtures of oligonucleotide drugs currently approved or in development contain many stereoisomers that exhibit low activity, thereby reducing the overall potency of the bulk mixture, and a small number of hyperfunctional molecules. Identification of the most active stereoisomers would provide a major step forwards in oligonucleotide drug development, allowing for lower doses with more efficacious compounds. Notably, it has been suggested that a stereorandom mixture of $S_{\mathrm{p}}$ and $R_{\mathrm{p}}$ centres is required to balance stability and silencing activity ${ }^{115}$. Nucleotide stereochemistry has also been exploited for aptamer development (BOX 1). 
Nucleobase modification. Strategies to modify nucleobase chemistry are also being investigated. For example, pyrimidine methylation (5-methylcytidine and 5-methyluridine/ribothymidine) (FIG. 3) has the effect of increasing the oligonucleotide melting temperature by $\sim 0.5^{\circ} \mathrm{C}$ per substitution ${ }^{97}$, and has been commonly incorporated into ASOs (such as those under development by Ionis Pharmaceuticals).

In addition, abasic nucleotides (that is, nucleotides lacking a nucleobase) (FIG. 2) have been used to abrogate miRNA-like silencing while maintaining on-target slicer activity ${ }^{116}$ and for allele-specific silencing of mutant HTT and ATXN3 transcripts ${ }^{117}$.

Terminal modification. Phosphorylation of the $5^{\prime}$ terminus of the siRNA guide strand is essential for activity, as this group makes an important contact in the MID domain of AGO2 (REFS ${ }^{118,119}$ ). Removal of this terminal phosphate group by cellular phosphatases therefore has the effect of reducing siRNA potency. The addition of a 5'-(E)-vinylphosphonate modification acts as a phosphate mimic that is not a phosphatase substrate. This modification also protects against exonuclease degradation and enhanced silencing in vivo ${ }^{120}$. Similarly, terminal inverted abasic ribonucleotides have been used to block exonuclease activity ${ }^{121}$. The conjugation of delivery-promoting moieties to oligonucleotide termini is discussed below.

Ribose sugar modification. Oligonucleotides are frequently modified at the $2^{\prime}$ position of the ribose sugar. Combinations of DNA (2'-deoxy) and RNA bases are critical to the activity of gapmer ASOs (that is, for generating RNase $\mathrm{H}$ substrate heteroduplexes), and are utilized on the $3^{\prime}$ termini of some siRNA designs in order to confer nuclease resistance ${ }^{35}$.

Similarly, 2'-O-methyl (2'-OMe), 2'-O-methoxyethyl $\left(2^{\prime}\right.$-MOE) and $2^{\prime}$-Fluoro ( $2^{\prime}$-F) (FIC. 3) are among the most commonly used $2^{\prime}$ substituents. These modifications increase oligonucleotide nuclease resistance by replacing the nucleophilic 2'-hydroxyl group of unmodified RNA, leading to improved stability in plasma, increased tissue half-lives and, consequently, prolonged drug effects. Furthermore, these modifications also enhance the binding affinity of the oligonucleotide for complementary RNA by promoting a 3'-endo pucker conformation (RNA-like) of the ribose ${ }^{122,123}$. These 2 -ribose modifications are not compatible with RNase $\mathrm{H}$ activity, meaning they are typically used for steric block oligonucleotides, or for the flanking sequences in gapmer ASOs. Although 2' substitutions that enhance binding affinity are not improvements in delivery per se, they can compensate for limited drug bioavailability as the fraction of the injected dose that reaches its intended target is more active.

For steric block and gapmer ASOs, the oligonucleotide simply needs to bind to its cognate target (and support RNase H cleavage with a DNA gap in the case of the latter). For siRNAs, the situation is more complex as the oligonucleotides must maintain the capacity for loading into an AGO2 protein and support slicer cleavage. However, extensive chemical modification has been reported using 2'-OMe and 2'-F chemistries ${ }^{124}$, and active siRNAs have been generated in which every single 2'-hydroxyl is modified, suggesting that the RNAi machinery is remarkably tolerant of chemical modification $^{125,126}$. Furthermore, full chemical modification has been shown to be highly important for the activity of siRNA bioconjugates ${ }^{127}$ (discussed below). Conversely, the introduction of 2'-ribose modifications at certain specific positions can ablate RISC loading and silencing activity. This phenomenon has been exploited in order to inactivate the passenger strand of the siRNA duplex, and thereby minimize its potential to mediate off-target silencing effects ${ }^{128}$.

2'-MOE modifications are not typically incorporated into siRNA designs (the one exception being single-stranded siRNAs ${ }^{42}$ ). Alnylam Pharmaceuticals has developed two patterns of siRNA chemical modification that form the basis for many of the products in their development pipeline ${ }^{129}$. The first is standard template chemistry, which consist of an alternating pattern of 2'-F and 2'-OMe modifications at all ribose positions. This design was shown to increase siRNA potency by more than 500-fold relative to the unmodified PO siRNA in some cases ${ }^{126}$. The now-discontinued drug revusiran (TABLE 2), targeting TTR for transthyretin amyloidosis, is an example of a standard template chemistry siRNA design ${ }^{130}$. The second approach is termed enhanced stability chemistry, in which siRNAs contain a greater proportion of 2'-OMe than standard template chemistry siRNAs and also incorporate PS linkages at the two internucleotide bridges at the $3^{\prime}$ terminus of the guide strand and the $5^{\prime}$ termini of both strands ${ }^{129,131}$. The approved Alnylam drug givosiran (FIG. $1 \mathrm{~h}$ ) is an example of an enhanced stability chemistry siRNA design.

2'-OMe modifications can also abrogate the immune responses that can be induced by ASOs, siRNAs and CRISPR guide RNAs (BOX 2). These oligonucleotide drugs have the potential to stimulate immune reactions in both sequence and chemistry-dependent ways via cellular pattern recognition receptors located in the cytoplasm or endosome ${ }^{121,132,133}$. Specifically, the Toll-like receptors can induce the interferon response: TLR3 recognizes double-stranded RNA motifs; TLR7 and TLR8 recognize single-stranded RNA; and TLR9 recognizes unmethylated CpG dinucleotides ${ }^{134,135}$. Similarly, the RIG-I and PKR systems also recognize double-stranded RNA in the cytoplasm ${ }^{136}$. Some of these immunogenic effects of siRNAs can be ablated by the inclusion of 2'-OMe modifications at key positions ${ }^{137,138}$. Conversely, incorporation of 5'-triphosphate-modified oligonucleotides ${ }^{139}$, or conjugation with CpG motif-containing TLR9 agonists $^{140,141}$, and other chemical modifications ${ }^{142}$ have been used to generate therapeutic immunostimulatory oligonucleotides.

Bridged nucleic acids. Bridged nucleic acids (BNAs) are types of nucleotide in which the pucker of the ribose sugar is constrained in the $3^{\prime}$-endo conformation via a bridge between the $2^{\prime}$ and $4^{\prime}$ carbon atoms. The most commonly used variations are locked nucleic acid (LNA) ${ }^{143,144}$, $2^{\prime}, 4^{\prime}$-constrained 2'-O-ethyl (constrained ethyl) BNA (cEt) and, to a lesser extent, $2^{\prime}-O, 4^{\prime}-C$-ethylene-bridged 
nucleic acid (ENA) ${ }^{145,146}$ (FIG. 3). BNAs enhance both nuclease stability and the affinity of the oligonucleotide for target RNA (typically by an increase of $3-8^{\circ} \mathrm{C}$ in melting temperature per modified nucleotide in the case of LNA $^{147}$ ). BNA modifications have therefore been incorporated into the flanking regions of gapmers to improve target binding. As such, cEt-flanking 3-10-3 gapmers are more efficacious than the MOE 5-10-5 equivalents ${ }^{98}$. Conversely, BNA nucleotides are not compatible with RNase H-mediated cleavage and so are excluded from the DNA gap region. LNA modifications have also been utilized in steric block ASOs, such as miRNA inhibitors. For example, miravirsen and cobomarsen (discussed above) are both full PS ASO mixmers containing DNA and LNA modifications distributed throughout their sequences. Conversely, tiny LNAs are short (8mer) fully LNA-modified oligonucleotides designed to simultaneously inhibit multiple members of an miRNA family (as these may execute redundant physiological functions) through complementarity to the miRNA seed sequence that is common between family members ${ }^{148}$.

Alternative chemistries. Whereas the majority of oligonucleotides are derived from RNA or DNA, other chemistries have been developed that differ substantially from these natural archetypes. PMO (phosphorodiamidate morpholino oligonucleotide) is a charge-neutral nucleic acid chemistry in which the five-membered ribose heterocycle is replaced by a six-membered morpholine ring ${ }^{149,150}$ (FIG. 3). Sarepta Therapeutics is developing PMO-based steric block ASOs for exon skipping in the context of DMD (TABLE 2). To date, two PMO drugs have been approved by the FDA, eteplirsen and golodirsen (FIG. 1d,e; TABLE 1), which target exons 51 and 53 of the dystrophin mRNA, respectively. Sarepta is also developing further ASO products based on the same chemistry targeting dystrophin exons $43,44,45$ (casimersen), 50, 52 and 55 (Sarepta Therapeutics' pipeline) (TABLE 2). Additionally, Nippon Shinyaku Pharma recently published encouraging data on a rival exon 53-targeting PMO (viltolarsen, NS-065/NCNP-01) ${ }^{151}$ and received marketing authorization in Japan in March 2020 (see Related links) (TABLE 2).

Notably, PMO backbone linkages contain chiral centres, meaning that PMO drugs are necessarily racemic mixtures. In contrast with PS modifications described above, the effects of defined PMO stereochemistry have not been explored to date.

Another strategy that has been explored is the use of peptide nucleic acid (PNA), a nucleic acid mimic in which a pseudo peptide polymer backbone substitutes for the PO backbone of DNA/RNA ${ }^{152,153}$ (FIG. 3). As both PMOs and PNAs are uncharged nucleic acid molecules, they can be covalently conjugated to charged delivery-promoting moieties such as cell-penetrating peptides (CPPs) (discussed below). Conversely, a disadvantage of these chemistries is that both PMO and PNA interact minimally with plasma proteins, meaning that they are rapidly cleared via urinary excretion.

The use of a constrained DNA analogue that increases the stability of RNA target-oligonucleotide duplexes by $2.4{ }^{\circ} \mathrm{C}$ per modification, known as tricyclo-DNA
(tcDNA) (FIG. 3), is also being investigated ${ }^{154}$. Interestingly, systemically administered tcDNA ASOs were shown to exhibit activity in the brain, suggesting that these molecules have the capacity to deliver oligonucleotides across the $\mathrm{BBB}^{155}$. Given the non-natural structures of $\mathrm{PMO}$, PNA and tcDNA, these chemistries are less suitable to RNase $\mathrm{H}$ and RNAi applications but have instead been used in steric block oligonucleotides, and for splice correction in particular ${ }^{155-157}$. However, tcDNA has recently been incorporated into the flanking sequences of a gapmer designed to silence mutant HTT transcripts ${ }^{158}$.

A final example is the development of a novel short interfering ribonucleic neutral (siRNN) chemistry ${ }^{159}$. These siRNN molecules contain a modified phosphotriester structure that neutralizes the charge of the equivalent unmodified PO/PS linkages, and thereby promotes their uptake in recipient cells. siRNNs act as prodrugs, which are converted to classical siRNAs by thioesterases in the cytoplasm ${ }^{159}$.

Bioconjugation. The delivery potential of ASOs and siRNAs can be enhanced through direct covalent conjugation of various moieties that promote intracellular uptake, target the drug to specific cells/tissues or reduce clearance from the circulation. These include lipids (for example, cholesterol that facilitates interactions with lipoprotein particles in the circulation $)^{160-162}$, peptides (for cell targeting and/or cell penetration) $)^{5,163-167}$, aptamers $^{168}$, antibodies ${ }^{9,169}$ and sugars (for example, $N$-acetylgalactosamine (GalNAc) $)^{170,171}$. Bioconjugates constitute distinct, homogeneous, single-component molecular entities with precise stoichiometry, meaning that high-scale synthesis is relatively simple and their pharmacokinetic properties are well defined. Furthermore, bionconjugates are typically of small size (relative to nanoparticle approaches, discussed below), meaning that they generally exhibit favourable biodistribution profiles (on account of being able to reach tissues beyond those with discontinuous or fenestrated endothelia).

For siRNAs there are four termini to which conjugates could potentially be attached. However, conjugation to the $5^{\prime}$ terminus of the guide strand is avoided as this terminal phosphate makes specific contacts with side-chain residues within the MID domain of AGO2 that are required for RNAi activity ${ }^{118,119}$. Conjugation to the passenger strand is generally preferred so as not to encumber the on-target silencing activity of the guide strand and, conversely, to diminish the off-target gene silencing potential of the passenger strand. Conjugates can be designed such that they are disassembled following cellular entry. This can be achieved by using acid-labile linkers that are cleaved in the endosome, disulfide linkers that are reduced in the cytoplasm or Dicer substrate-type siRNA designs ${ }^{172}$.

A common theme in oligonucleotide bioconjugation approaches is the promotion of interactions between the conjugate and its corresponding cell surface receptor protein, leading to subsequent internalization by receptor-mediated endocytosis. The interaction of bioconjugates with cell type-associated receptors thereby enables targeted delivery to specific tissues, or cell types 
a<smiles>CC(C)C=CC[C@@H]1CC(O)CN1C(=O)CCCCCNC(=O)OC1CC[C@@]2(C)C(=CCC3C4CC[C@H]([C@@H](C)CCCC(C)C)[C@]4(C)CCC32)C1</smiles>

C

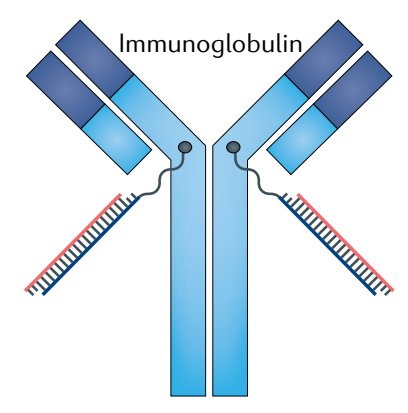

Antibody-siRNA

b

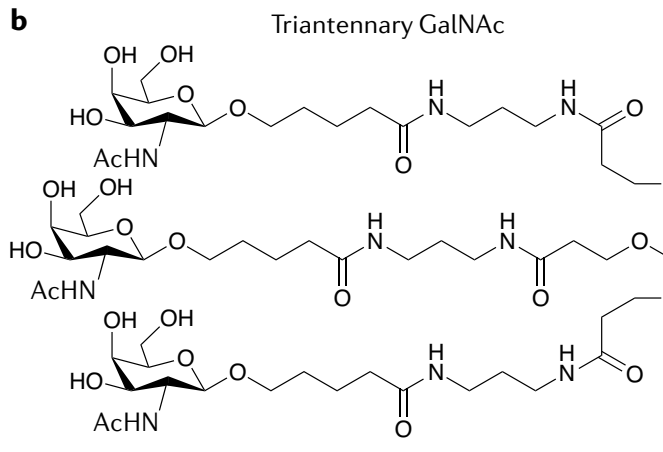

e

Peptide-ASO

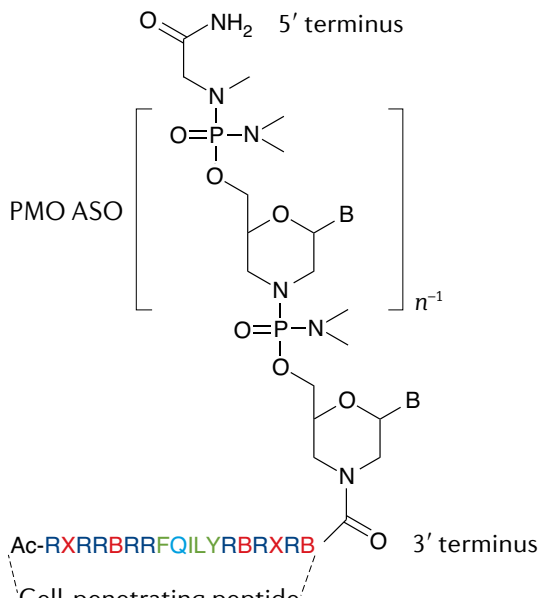

Cell-penetrating peptide

B $\beta$-Alanine $\times$ Aminohexanoic acid

h

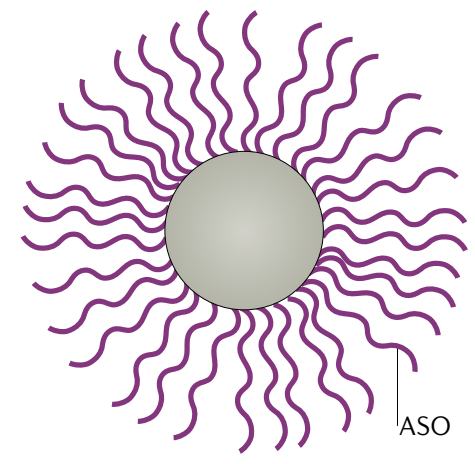

Spherical nucleic acid f

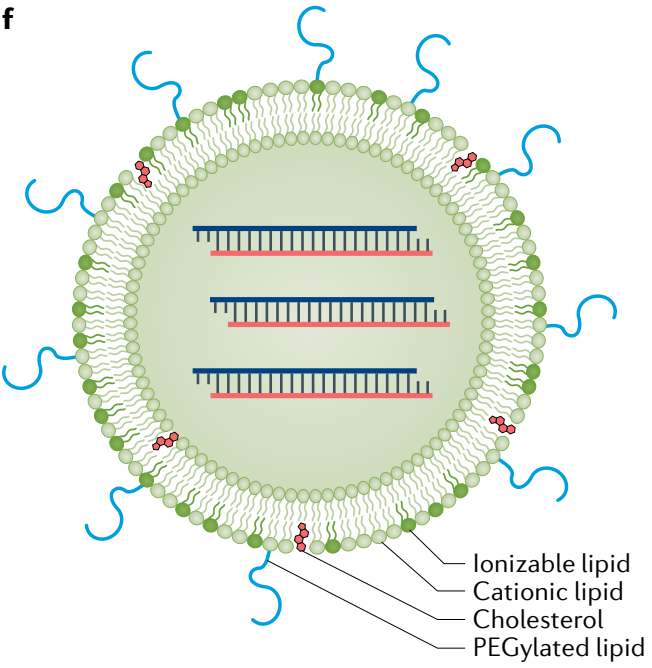

Stable nucleic acid lipid particle

\section{.}

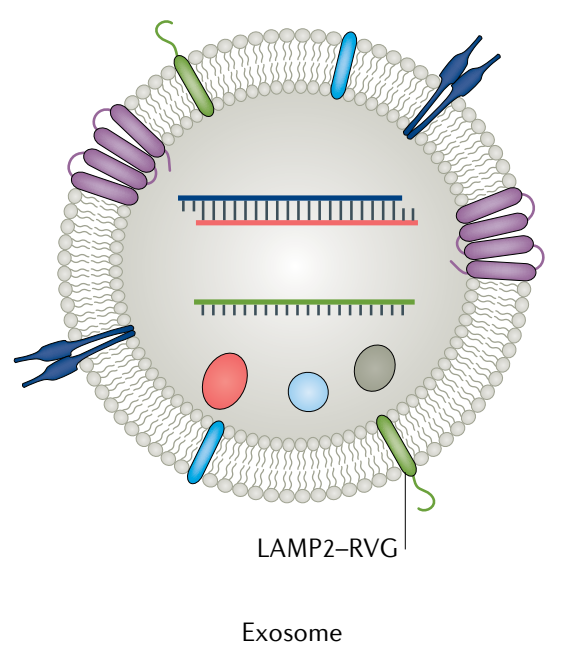

i

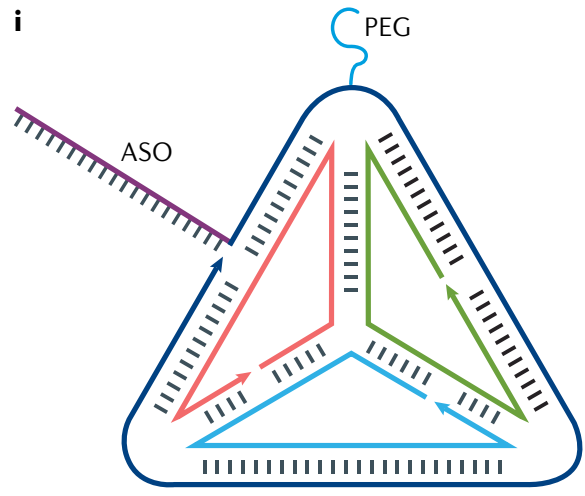

DNA cage 
4 Fig. 4 | Oligonucleotide delivery strategies. Schematics of various delivery strategies for small interfering RNAs (siRNAs) and antisense oligonucleotides (ASOs). a | LipidsiRNA conjugate wherein cholesterol is conjugated to the 3 'terminus of the passenger strand. $\mathbf{b}$ | Triantennary $\mathrm{N}$-acetylgalactosamine (GalNAc) moiety conjugated to an ASO. c|Antibody-siRNA conjugate. Oligonucleotides can be attached to the antibody or Fab fragment using click chemistry or thiol-maleimide linkages. $\mathbf{d} \mid$ Aptamer-siRNA conjugate. In vitro transcription can be used to generate a chimaeric aptamer-passenger strand as a single molecule. e | Peptide-ASO conjugate. The example is a PMO (phosphorodiamidate morpholino oligonucleotide) conjugated to a cell-penetrating peptide (Pip-9b2) ${ }^{209}$. f | Stable nucleic acid lipid particle encapsulating siRNAs. g| Engineered exosome with the brain-targeting rabies virus glycoprotein (RVG) peptide displayed on the outer surface ${ }^{255}$. The exosome consists of a membrane containing lipids and proteins derived from the donor cell. The exosome also contains therapeutic cargo (for example, siRNA) and proteins and nucleic acids (for example, microRNA) derived from the donor cell. $\mathbf{h} \mid$ Spherical nucleic acid nanoparticle consisting of a gold core coated in densely packed ASOs attached by metal-thiol linkages. i | Self-assembled DNA cage tetrahedron nanostructure. Oligonucleotide therapeutics (for example, siRNAs and ASOs) can be incorporated into the design of the DNA cage itself. Additional targeting ligands and polyethylene glycol (PEG) can be further conjugated to the nanostructure. LAMP2, lysosome-associated membrane protein 2; Pip, PMO/peptide nucleic acid internalization peptide. Part $\mathbf{d}$ shows a schematic of the PSMA (prostate-specific membrane antigen) aptamer adapted from REF. ${ }^{168}$, Springer Nature Limited. the adrenal glands, ovary, kidney and small intestine ${ }^{160}$. Accordingly, endocytosis of cholesterol siRNAs was shown to be mediated by scavenger receptor-type B1 (SCARB1, SR-B1) or LDL receptor (LDLR) for HDL and LDL particles, respectively ${ }^{160}$. In vivo association of siRNAs with the different classes of lipoprotein is governed by their overall hydrophobicity, with the more hydrophobic conjugates preferentially binding to LDL and the less lipophilic conjugates preferentially binding to $\mathrm{HDL}^{176}$.

GalNAc conjugates. GalNAc is a carbohydrate moiety that binds to the highly liver-expressed asialoglycoprotein receptor 1 (ASGR1, ASPGR) with high affinity $\left(K_{\mathrm{d}}=2.5 \mathrm{nM}\right)^{177}$ and facilitates the uptake of PO ASOs ${ }^{178,179}$ and siRNAs into hepatocytes by endocytosis ${ }^{129,170,171,180}$. ASGR1 is very highly expressed in the liver, and is rapidly recycled to the cell membrane, making it an ideal receptor for effective liver-targeted delivery. The interaction between GalNAc and ASGR1 is pH-sensitive, such that dissociation of the receptor and oligonucleotide conjugate occurs during acidification of the endosome ${ }^{129}$. The GalNAc moiety is subsequently subject to enzymatic degradation that liberates the oligonucleotide ${ }^{179}$. GalNAc-conjugated ASOs are preferentially delivered to hepatocytes in vivo, whereas unconjugated ASOs are primarily detected in non-parenchymal liver cells ${ }^{179}$.

Typically, a triantennary GalNAc structure (FIG. 4b) is used as the conjugated moiety, although there are other structural variants ${ }^{129,181}$. GalNAc conjugation enhanced ASO potency by $\sim$-fold in mouse, specific to the liver ${ }^{179}$, and by $\sim 30$-fold in human patients ${ }^{182}$. As such, GalNAc conjugation is now one of the leading strategies for delivering experimental oligonucleotide drugs currently in development, given its high liver silencing potential, small size relative to nanoparticle complexes, defined chemical composition and low cost of synthesis. In particular, GalNAc conjugation features heavily in the drug development pipelines of several pharma companies, most notably Alnylam, who are developing drugs for the treatment of diseases such as haemophilia A and B and primary hyperoxaluria type 1 (TABLE 2). Furthermore, a GalNAc-conjugated siRNA, givosiran (developed by Alnylam), received FDA approval in November 2019 (TABLE 1). Givosiran is a GalNAc-conjugated, blunt-ended, enhanced stability chemistry siRNA duplex targeting 5 '-aminolevulinate synthase 1 (ALAS1) for the treatment of acute hepatic porphyria $^{183}$. Similarly, inclisiran, a second GalNAcconjugated siRNA containing 2'-F, 2'-OMe and PS modifications (developed by Alnylam/The Medicines Company and acquired by Novartis ${ }^{184}$ ), is in late-stage clinical trials for the treatment of familial hypercholesterolaemia (TABLE 2). Inclisiran targets PCSK9 (proprotein convertase subtilisin/kexin type 9 ), which is a circulating factor that negatively regulates expression of LDLR and is primarily expressed in the liver. Hepatic PCSK9 knockdown therefore increases the availability of LDLR to remove LDL cholesterol from the circulation $^{185,186}$. Subcutaneous injection of inclisiran resulted in long-term downregulation of circulating PCSK9 and LDL cholesterol ( $\sim 6$ months) suggesting 
that an infrequent treatment regimen may be a sufficient lipid-lowering strategy ${ }^{180,187}$.

Numerous additional pharmaceutical companies namely Dicerna Pharmaceuticals, Silence Therapeutics, Arbutus Biopharma and Arrowhead Pharmaceuticals are also developing GalNAc-conjugated oligonucleotide products (TABLE 2).

Antibody and aptamer conjugates. Although there is a plethora of technologies capable of delivering nucleic acids to hepatic cells, there is still a need for strategies that can target cell surface receptors specific to other tissues. Antibodies have been used as delivery vehicles for other kinds of drugs ${ }^{188}$, although their utility for oligonucleotide delivery is still in the early stages of development. Specific interactions between an antibody and a cell surface receptor have the potential to enable delivery to tissues and/or cell subpopulations that are not accessible using other technologies. Various receptors have been successfully targeted for siRNA delivery (FIG. 4d), including the HIV gp160 protein ${ }^{169}$, HER2 (REF. ${ }^{189}$ ), CD7 ( $\mathrm{T}$ cell marker) ${ }^{190}$, CD71 (transferrin receptor, highly expressed in cardiac and skeletal muscle) ${ }^{191}$ and TMEFF2 (REF. ${ }^{192}$ ). Similarly, ASOs have also been conjugated with antibodies against CD44 (a neural stem cell marker), EPHA2 and EGFR ${ }^{193}$. In these cases, the ASO was delivered as a duplex with a DNA carrier strand to which the antibody was attached via click chemistry ${ }^{194}$. Such a design allows the DNA passenger to be degraded after cellular entry, thereby releasing the ASO from the complex ${ }^{194}$. Antibody-siRNA and antibody-ASO conjugates targeting tissues such as skeletal muscle are currently being developed by Avidity Biosciences (TABLE 2) and Dyne Therapeutics, respectively.

Similarly, the conjugation of therapeutic oligonucleotides to nucleic acid aptamers (BOX 1) has also been explored for enhancing delivery of siRNAs and ASOs to specific target cells $s^{168,195,196}$. Aptamers can be considered 'chemical antibodies' that bind to their respective target proteins with high affinity, but present numerous advantages over antibodies as they are simple and inexpensive to manufacture (that is, by chemical synthesis), are smaller in size and exhibit lower immunogenicity ${ }^{197}$.

Peptide conjugates. Peptides are an attractive source of ligands that may confer tissue/cell-targeting, cell-penetrating (that is, CPPs) or endosomolytic properties onto therapeutic oligonucleotide conjugates. CPPs (also known as protein transduction domains) are short (typically $<30$ amino acids) amphipathic or cationic peptide fragments that are typically derived from naturally occurring protein translocation motifs (as in the case of HIV-TAT (transactivator of transcription protein), Penetratin 1 (homeodomain of the Drosophila Antennapedia protein) and Transportan (a chimeric peptide consisting of part of the galanin neuropeptide fused to the wasp venom, mastoparan)) or are based on polymers of basic amino acids (that is, arginine and lysine) ${ }^{198}$. One of the most promising applications of CPPs is their direct chemical conjugation to charge-neutral ASO chemistries, such as PMO and PNA. Several groups have pioneered the use of peptide-PMO (PPMO) conjugates
(FIG. 4e) for the treatment of various diseases, most notably for dystrophin splice switching in the context of DMD. Early PPMO dystrophin exon skipping studies demonstrated efficacy using (RXR) ${ }_{4}-\mathrm{PMO}^{199,200}$ and the ' $\mathrm{B}$ ' peptide (with sequence (RXRRBR) ${ }_{2} \mathrm{XB}$ ) ${ }^{199,201}$, where $\mathrm{X}$ and $\mathrm{B}$ are 6 -aminohexanoic acid and $\beta$-alanine spacer residues, respectively. The spacer residues are important for the optimal positioning of the charged arginine side chains ${ }^{202,203}$. This approach was further modified by generating a chimeric peptide consisting of B peptide fused with a muscle-targeting peptide (MSP) ${ }^{204}$. The resulting B-MSP-PMO conjugates demonstrated further dystrophin restoration efficacy in the $m d x$ mouse model of DMD (although the relative arrangement of the peptide constituents was found to be important, with MSP-B-PMO exhibiting low activity ${ }^{204,205}$. Exon skipping activity has also been reported when PMOs were conjugated to a different muscle-targeting peptide (M12) identified by phage display, although activity in the heart was minimal ${ }^{206}$. Subsequently, several series of peptides known as 'Pip's (PMO/PNA internalization peptide) consisting of $\mathrm{R}, \mathrm{B}$ and $\mathrm{X}$ amino acids with an internal core containing hydrophobic residues have been developed ${ }^{164,207-209}$. Current-generation Pip-PMO conjugates (FIG. 4 e) are much more potent than naked PMO in dystrophic animal models and, importantly, reach cardiac muscle (a tissue critical to the lethality of DMD) after systemic delivery ${ }^{164,209-211}$. The PPMO hydrophobic core is required for cardiac delivery, but can itself be scrambled, inverted, or individual residues substituted with only minimal changes to efficacy ${ }^{164,209}$. A major challenge for PPMO technology is toxicity, with evidence of renal damage in both rat (at very high doses) and cynomolgus monkey studies using arginine-rich CPP-PMOs ${ }^{212,213}$. Notably, the arginine content of the $\mathrm{CPP}$ is correlated with both exon skipping activity and nephrotoxicity ${ }^{209}$, and so current research efforts are directed towards the optimization of peptide chemistry to mitigate renal toxicity without compromising splice correction efficacy. Sarepta Therapeutics is developing SRP-5051, a PPMO designed to skip dystrophin exon 51 (TABLE 2). Additionally, PepGen Ltd is commercializing PPMO technology (TABLE 2).

PPMO uptake is energy dependent and appears to involve distinct endocytic pathways in skeletal and cardiac muscle cells ${ }^{214}$. It has been reported that treatment of chloroquine can enhance PPMO activity, suggesting that many conjugate molecules may not escape the endolyosomal compartment ${ }^{215}$. PPMOs have also been shown to spontaneously form micelles of defined sizes and surface charge, meaning that they are more readily taken up by endocytosis, in part mediated by scavenger receptors $^{104}$.

PPMO technology has also been demonstrated to be effective for targeting CUG repeat-expanded transcripts in the context of myotonic dystrophy type I (DM1) (whereas naked PMO was completely ineffective) ${ }^{5}$, for splice correction to restore BTK expression for the treatment of X-linked agammaglobulinaemia ${ }^{216}$ and for delivery to the CNS in animal models of spinal muscular atrophy ${ }^{211}$. Similarly, brain delivery (to the cerebellum and Purkinje cells in particular) of an arginine-rich 
CPP-PMO conjugate after systemic delivery has also been demonstrated ${ }^{217}$.

PPMOs are also promising antibacterial (as they are capable of traversing the bacterial cell wall) and antiviral agents. Intranasal administration of (RXR) peptide-PMO conjugates targeting an essential bacterial gene $a c p P$ in murine infection models was shown to be bactericidal and increased survival ${ }^{218}$. Further, arginine-rich peptide-PMO conjugates were shown to exert protective effects in murine viral infection models of SARS-CoV ${ }^{219,220}$ and Ebola ${ }^{221}$.

Conjugation of peptides to charged-backbone oligonucleotides has been explored to a much lesser extent, as charge-charge interactions between the constituents complicate synthesis and purification, and conjugates may have the potential to self-aggregate. Nevertheless, there are a few examples of such conjugates. It was recently demonstrated that conjugation of an ASO gapmer to a ligand for the glucagon-like peptide 1 receptor (GLP1R) conferred targeted gene silencing in pancreatic $\beta$-cells ${ }^{166}$, the pancreas being a particularly challenging organ to deliver to. In this case, the targeting moiety was a 40-amino acid peptide consisting of a modified GLP1 sequence covalently conjugated to the ASO via the carboxy terminus. Peptide conjugation has also been explored for siRNA delivery. For example, the cyclic RGD peptide (recognized by $\alpha v \beta 3$ integrin receptors) has successfully been used to deliver anti-VEGFR2 siRNA conjugates to mouse tumours ${ }^{167}$. Similarly, the CPPs TAT (48-60) and penetratin have been utilized as siRNA conjugates for delivery to the lung via the intratracheal route $^{222}$. Although modest silencing of the target gene was observed, administration of the unconjugated peptides alone also exhibited a repressive effect. Furthermore, treatment with the penetratin-siRNA conjugates was associated with the release of pro-inflammatory markers TNF, IL-12 p40 and IFNa ${ }^{222}$. These observations highlight that potential peptide-mediated non-specific effects on gene expression and innate immune activation must be carefully considered.

Nanocarriers. Advances in nanotechnology and material science offer advantages and potential solutions to the challenge of oligonucleotide drug delivery, in particular the requirements for crossing biological barriers and transmembrane intracellular delivery. The major advantages of nanoparticle delivery systems include bespoke optimization of nanoparticle biophysical (for example, size, shape and chemical/material composition) and biological (for example, ligand functionalization for targeting) properties, allowing for highly tailored delivery platforms. A wide range of nanocarriers for nucleic acid drug delivery are at various stages of development, including non-covalent complexation with cationic polymers (for example, polyethylenimine) ${ }^{223}$, dendrimers ${ }^{224,225}$, CPPs (for example, MPG-8 (REF. ${ }^{226}$ ), PepFect6 (REF. ${ }^{227}$ ), RVG-9R ${ }^{228}$, and Xentry-KALA ${ }^{229}$ ) and inorganic methods (for example, calcium phosphate nanoparticles $)^{230}$. Below, we focus on lipid-based formulations for oligonucleotide delivery and emerging novel approaches including endogenously derived exosomes, SNAs and self-assembling DNA nanostructures.
Lipoplexes and liposomes. Formulation with lipids is one of the most common approaches to enhancing nucleic acid delivery. Mixing polyanionic nucleic acid drugs with lipids leads to the condensing of nucleic acids into nanoparticles that have a more favourable surface charge, and are sufficiently large $(\sim 100 \mathrm{~nm}$ in diameter) to trigger uptake by endocytosis. Lipoplexes are the result of direct electrostatic interaction between polyanionic nucleic acid and the cationic lipid, and are typically a heterogeneous population of relatively unstable complexes. Lipoplex formulations need to be prepared shortly before use, and have been successfully used for local delivery applications ${ }^{228}$. By contrast, liposomes comprise a lipid bilayer, with the nucleic acid drug residing in the encapsulated aqueous space. Liposomes are more complex (typically consisting of cationic or fusogenic lipids (to promote endosomal escape $^{231}$ ) and cholesterol PEGylated lipid) and exhibit more consistent physical properties with greater stability than lipoplexes ${ }^{232}$. For example, some lipid nanoparticles (LNPs), also known as stable nucleic acid lipid particles (FIG. 4f), are liposomes that contain ionizable lipid, phosphatidylcholine, cholesterol and PEG-lipid conjugates $^{233,234}$ in defined ratios and have been successfully utilized in multiple instances. Landmark examples are the silencing of hepatitis $B$ virus and $A P O B$ by siRNAs in preclinical animal studies ${ }^{137,235}$ and, more recently, the approval of patisiran, an siRNA that is delivered as an LNP formulation ${ }^{236}$. Encapsulation of nucleic acid cargos provides a means of protection from nuclease digestion in the circulation and in the endosome. Additionally, ionizable LNPs also associate with APOE, which further facilitates liver uptake via LDLR-mediated endocytosis ${ }^{237}$. Similarly, LNPs containing lipidoid or lipid-like materials have demonstrated robust siRNA-mediated silencing in rodents ${ }^{238,239}$ and non-human primates ${ }^{240}$.

A disadvantage of LNPs is that their delivery is primarily limited to the liver and reticuloendothelial system as the sinusoidal capillary epithelium in this tissue provides spaces large enough to allow the entry of these relatively large nanoparticles ${ }^{137,241,242}$. However, local delivery of LNPs has been used to successfully deliver siRNAs to the CNS after intracerebroventricular injection ${ }^{243}$. Conversely, the large size of nanoparticles is advantageous as it essentially precludes renal filtration ${ }^{244}$ and permits delivery of a higher payload.

LNPs can be further functionalized with peptides ${ }^{245}$, $\mathrm{PEG}^{246}$ or other ligands that confer cell-specific targeting (for example, GalNAc (hepatocytes) ${ }^{234,237}$, anisamide (lung tumours) ${ }^{247}$, strophanthidin (various tumours) ${ }^{248}$ and vitamin A (hepatic stellate cells) ${ }^{249}$ ). Notably, an increase in the complexity of LNPs complicates manufacture and may increase their toxicity, which is a major concern that may limit their clinical utility. For example, LNP siRNA particles (such as patisiran) require premedication with steroids and antihistamines to abrogate unwanted immune reactions ${ }^{250}$.

Exosomes. An area of nanotechnology that is gaining interest is based on the application of natural biological nanoparticles known as exosomes (a class of 
Multivesicular bodies

Membrane-bound

compartments within cells that contain intraluminal vesicles

that form as a consequence of inward budding of the

multivesicular body membrane. When multivesicular bodies fuse with the plasma membrane, their intraluminal vesicles are released into the extracellular space and are now considered exosomes. extracellular vesicle). Exosomes are heterogeneous, lipid bilayer-encapsulated vesicles approximately $100 \mathrm{~nm}$ in diameter that are generated as a result of the inward budding of the multivesicular bodies ${ }^{251,252}$. Exosomes are thought to be released into the extracellular space by all cells, where they facilitate intercellular communication via the transfer of their complex macromolecular cargoes (that is, nucleic acids, proteins and lipids) ${ }^{253,254}$. Exosomes present numerous favourable properties in terms of oligonucleotide drug delivery: exosomes are capable of traversing biological membranes, such as the $\mathrm{BBB}^{255}$; the presence of the marker protein $\mathrm{CD} 47$ protects exosomes from phagocytosis, thereby increasing their circulation time relative to liposomes ${ }^{256}$; exosomes are considered non-toxic and have been safely administered to patients with graft-versus-host disease ${ }^{257}$; exosomes have the potential to be produced in an autologous manner; exosomes from some sources have been shown to have inherent pro-regeneration and anti-inflammatory properties that may augment the effects of therapeutic oligonucleotide delivery ${ }^{258,259}$; and engineered exosomes can serve as a modular platform whereby combinations of therapies and/or targeting moieties can be deployed.

A major challenge for exosome therapeutics is the efficient loading of therapeutic oligonucleotide cargo. Vesicles can be loaded either endogenously (for example, by overexpression of the cargo in the producer cell line ${ }^{260}$ ) or exogenously (for example, by electroporation ${ }^{255,256,261}$, sonication ${ }^{262}$, co-incubation with cholesterol-conjugated siRNAs ${ }^{263,264}$ and so forth). The loading of exosomes with splice-switching PMOs has been achieved via conjugation with the CP05 peptide, which binds to CD63 (a marker commonly found on exosomes) so as to decorate the exosomes with PMO cargo $^{265}$.

The pattern of exosome biodistribution can be favourably altered through the display of surface ligands, such as peptides like rabies virus glycoprotein (RVG) to enhance brain penetration and facilitate delivery to cells within the nervous system ${ }^{255,266,267}$ (FIG. 4g) or GE11 that promotes binding to tumour cells by interacting with EGFR $^{268}$. Similarly, exosomes decorated with an RNA aptamer targeting PSMA (prostate-specific membrane antigen) were capable of delivering siRNAs to xenograft tumours and inducing tumour regression ${ }^{269}$.

Methods for the manufacture of therapeutic exosomes at high scale, including clinical grade, have been reported ${ }^{261}$. The use of mesenchymal stem cell lines (with the potential for immortalization) and culture in bioreactors enables large volumes of exosome-containing conditioned media to be generated. Subsequently, methods such as tangential flow filtration and size-exclusion liquid chromatography provide a scalable means of isolating therapeutic exosomes from these supernatants ${ }^{270}$. Therapeutic applications of engineered exosome technology are presently at an advanced preclinical stage for two companies: Codiak Biosciences and Evox Therapeutics.

Spherical nucleic acids. An alternative nanoparticlebased delivery strategy is the SNA approach. SNA particles consist of a hydrophobic core nanoparticle (comprising gold, silica or various other materials) that is decorated with hydrophilic oligonucleotides (for example, ASOs, siRNAs and immunostimulatory oligonucleotides) that are densely packed onto the surface via thiol linkages (FIG. 4h). In contrast to other nanoparticle designs, SNA-attached oligonucleotides radiate outwards from the core structure. While exposed, the oligonucleotides are protected from nucleolytic degradation to some extent as a consequence of steric hindrance, high local salt concentration, and through interactions with corona proteins ${ }^{271}$.

SNA particles carrying an siRNA targeting the anti-apoptotic factor Bcl2l12 were able to promote tumour apoptosis, reduce the tumour burden and extend survival in glioblastoma xenograft-bearing mice ${ }^{272}$. Importantly, this study demonstrated that SNAs have the potential to cross the BBB in both tumour-bearing mice (with impaired BBB integrity) and also in wildtype mice ${ }^{272}$, although the majority of SNA particles were deposited in the liver and kidneys. SNA particles have also been applied for topical delivery to skin keratinocytes in the context of diabetic wound healing (that is, GM3S-targeting siRNA) ${ }^{273}$ and psoriasis (that is, TNF-targeting ASO) ${ }^{274}$. SNA particles are currently being commercialized for oligonucleotide delivery applications by Exicure, Inc.

DNA nanostructures. DNA nanostructures, of which there are many varieties, have also been utilized for oligonucleotide delivery. These structures include DNA origami, whereby long DNA molecules are held in defined structures using short DNA 'staples' that enable a wide variety of complex shapes to be formed, including polygonal nanostructures such as DNA cages. DNA nanostructures typically self-assemble owing to base pairing complementarity of their constituent parts, and can be designed with precise geometries such that their physical properties (for example, size, flexibility and shape) can be fine-tuned in order to maximize their delivery potential. DNA nanostructures used for nucleic acid delivery applications will typically be modular structures that incorporate nucleic acid drugs (and targeting ligands such as aptamers) within the design of the structure itself. For example, DNA nanostructures have been designed that incorporate ASOs (FIG. 4i), siRNAs ${ }^{275}$ and immunostimulatory oligonucleotides ${ }^{276}$ displayed on the structure surface. A highly interesting property of DNA nanostructures is that they have been reported to not accumulate in the liver, and can be engineered to be small $(\sim 20 \mathrm{~nm})$, meaning extrahepatic delivery is possible. However, further reducing the size of DNA nanostructures without additional functionalization will likely result in enhanced renal filtration, and overall lower bioavailability ${ }^{275,277}$.

Stimuli-responsive nanotechnology. Stimuli-sensitive, activatable drug delivery nanotechnologies are emerging as oligonucleotide delivery solutions. Activatable CPP conjugates consist of an oligonucleotide covalently attached to a peptide that is folded into a hairpin structure. One half of the hairpin is arginine rich and positively charged, whereas the second half of the 
peptide is negatively charged and acts as a neutralizing inhibitory domain. The loop of the hairpin contains an enzymatic cleavage motif, allowing for activation of the conjugate when it reaches the desired site of action. Activation by matrix metalloproteinase 2 (MMP2) has been utilized to enable targeted delivery of siRNA to hepatoma cells in vitro ${ }^{278}$ and in xenograft tumours in vivo ${ }^{279}$. Similarly, dynamic polyconjugates consist of oligonucleotides conjugated to a scaffold that is linked to multiple delivery-assisting moieties. For example, an siRNA-dynamic polyconjugate consisting of a PBAVE scaffold polymer linked to PEG and NAG ( $N$-acetyl glucosamine, for liver targeting) moieties by acid-labile linkers induced potent gene silencing in mouse hepatocytes after intravenous administration ${ }^{280}$. The acidic environment of the endosome induces cleavage of the PEG and NAG groups, leading to exposure of the PBAVE tertiary amines, buffering of luminal $\mathrm{pH}$ and consequent endosomal escape ${ }^{280}$.

Even more complex 'smart' delivery vehicles are possible with DNA nanotechnology. For example, DNA origami has been used to generate a box that is opened and incorporates two structure-shifting aptamer 'locks'. When both aptamers interact with their target proteins, the lock opens and the DNA box changes conformation to release its contents ${ }^{281}$. This technology was utilized to deliver gold nanoparticles and antibody fragments but could potentially be modified for nucleic acid delivery. Such logic-gated delivery vehicles present numerous advantages as therapeutic payloads could be concentrated at the desired sites of action, leading to higher efficacy and reduced off-target effects. Technologies that can confer stimuli-responsiveness (for example, to $\mathrm{pH}$, temperature, redox state, enzymatic activity, magnetic fields and light) in nanoparticle drug delivery systems have been reviewed elsewhere ${ }^{282}$.

\section{Challenges and considerations}

The establishment of therapeutic platforms capable of delivering oligonucleotide drugs to specific organs or tissues will likely involve defined patterns of chemical modification, combined with conjugation/complexation strategies that confer predictable pharmacokinetic and pharmacodynamic properties, and well-understood mechanisms of action. In this manner, oligonucleotide therapeutics have the potential to extend the range of possible pharmaceutical targets and provide a means by which new drugs can be rapidly developed to meet unmet and emerging clinical needs, without the need to 'start from scratch'. Such a situation is exemplified by the case of milasen, an ASO drug designed as a personalized drug for a single patient suffering from Batten disease. Milasen was designed using the same full PS-2'-MOE design as nusinersen (FIG. 1 f; TABLE 1), an FDA-approved chemistry that distributes favourably throughout the CNS after intrathecal administration, resulting in a period of less than 1 year between identification of the patient's mutation and the first administration of the ASO drug ${ }^{1}$. Whether such a model of drug development is scalable to more patients remains to be seen, although the n-Lorem Foundation aims to use bespoke ASO drugs to treat patients with ultra-rare diseases, where conventional clinical trials are impossible. However, a key issue will be how the safety of different drug sequences using the same chemistry/delivery platform will be viewed by regulatory authorities.

Although LNPs and GalNAc conjugates offer excellent hepatic delivery in both preclinical and clinical studies, systemic delivery beyond the liver will require further investigation, innovation and development. In many cases, approved oligonucleotide drugs will likely be extremely, and possibly prohibitively, expensive. For example, nusinersen currently costs $\$ 750,000$ for the first year and $\$ 375,000$ in subsequent years. Similarly, the cost of eteplirsen is $\$ 300,000$ per annum. The costbenefit ratio for highly effective, life-changing medications such as nusinersen is likely to be favourable. By contrast, reimbursing the cost of eteplirsen, which has demonstrated very limited efficacy, will be much more difficult to justify. Importantly, drug costs should also be weighed against the cost of care for patients left untreated ${ }^{283}$. As oligonucleotide therapeutics are further combined with novel delivery modalities, such advances may compound the cost of materials. However, the improved efficacy and/or better targeted delivery afforded by such delivery technologies may mean that lower doses of drug can be administered, which may conversely reduce costs. Another key consideration is safety. While the immune-stimulating properties of standard nucleic acid modification chemistries are relatively well understood, the potential immunogenicity of delivery agent components or ligand conjugates may present additional challenges to safe and effective oligonucleotide drug delivery.

Given the large number of nucleic acid chemistries, delivery technologies and therapeutic modalities, direct head-to-head comparisons are unlikely to be possible in many cases. This issue is further compounded when considering the diversity of possible stereoisomers if chiral backbone modifications are used. The application of artificial intelligence and computer modelling may be one way to address these issues. Nevertheless, the approval of two oligonucleotide drugs with different mechanisms of action for the same indication (that is, inotersen and patisiran, a gapmer ASO and an siRNA, respectively; FIG. 1; TABLE 1) does at least allow for direct comparison of these approaches.

\section{Outlook}

Numerous recent, high-profile regulatory approvals have demonstrated that oligonucleotide drug delivery has now matured to the position of clinical utility for multiple diverse indications. The utility of highly optimized combinations of nucleic acid chemical modifications, conjugation to cell/tissue-targeting ligands and nanoparticle carrier systems has enhanced the efficiency of oligonucleotide drug delivery and/or is enabling therapeutic molecules to reach previously inaccessible target tissues. These developments provide hope that therapy for many rare or currently untreatable diseases will soon be possible through the use of precision genetic medicine.

Published online 11 August 2020 
1. Kim, J. et al. Patient-customized oligonucleotide therapy for a rare genetic disease. N. Engl. J. Med. 381, 1644-1652 (2019). This is the first study to utilize an oligonucleotide therapy tailored to a single patient.

2. Giorgio, E. et al. Allele-specific silencing as treatment for gene duplication disorders: proof-of-principle in autosomal dominant leukodystrophy. Brain 142, 1905-1920 (2019)

3. Southwell, A. L. et al. In vivo evaluation of candidate allele-specific mutant huntingtin gene silencing antisense oligonucleotides. Mol. Ther. 22, 2093-2106 (2014).

4. Miller, V. M. et al. Allele-specific silencing of dominant disease genes. Proc. Natl Acad. Sci. USA 100 , 7195-7200 (2003)

5. Klein, A. F. et al. Peptide-conjugated oligonucleotides evoke long-lasting myotonic dystrophy correction in patient-derived cells and mice. J. Clin. Invest. 129 4739-4744 (2019).

6. Crnković-Mertens, I., Semzow, J., Hoppe-Seyler, F. \& Butz, K. Isoform-specific silencing of the Livin gene by RNA interference defines Livin $\beta$ as key mediator of apoptosis inhibition in HeLa cells. J. Mol. Med. 84, 232-240 (2006)

7. Valencia-Serna, J. et al. siRNA-mediated BCR-ABL silencing in primary chronic myeloid leukemia cells using lipopolymers. J. Controlled Rel. 310, 141-154 (2019).

8. Wu, S. Y., Lopez-Berestein, G., Calin, G. A. \& Sood, A. K RNAi therapies: drugging the undruggable. Sci. Transl. Med. 6, 240ps7 (2014).

9. Tushir-Singh, J. Antibody-siRNA conjugates: drugging the undruggable for anti-leukemic therapy. Expert Opin. Biol. Ther. 17, 325-338 (2017)

10. Bobbin, M. L., Burnett, J. C. \& Rossi, J. J. RNA interference approaches for treatment of HIV-1 infection. Genome Med. 7, 50 (2015).

11. Hermann, T. \& Patel, D. J. Adaptive recognition by nucleic acid aptamers. Science 287, 820-825 (2000).

12. Knott, G. J. \& Doudna, J. A. CRISPR-Cas guides the future of genetic engineering. Science 361, 866-869 (2018).

13. Jackson, A. L. et al. Expression profiling reveals off-target gene regulation by RNAi. Nat. Biotechnol. 21, 635-637 (2003)

14. Jackson, A. L. et al. Widespread siRNA 'off-target' transcript silencing mediated by seed region sequence complementarity. RNA 12, 1179-1187 (2006).

15. Scacheri, P. C. et al. Short interfering RNAs can induce unexpected and divergent changes in the levels of untargeted proteins in mammalian cells. Proc. Natl Acad. Sci. USA 101, 1892-1897 (2004).

16. Persengiev, S. P., Zhu, X. \& Green, M. R. Nonspecific concentration-dependent stimulation and repressio of mammalian gene expression by small interfering RNAs (siRNAs). RNA 10, 12-18 (2004).

17. Doench, J. G., Petersen, C. P. \& Sharp, P. A. siRNAs can function as miRNAs. Genes Dev. 17, 438-442 (2003).

18. Grimm, D. et al. Fatality in mice due to oversaturation of cellular microRNA/short hairpin RNA pathways. Nature 441, 537-541 (2006).

19. Wu, H. et al. Determination of the role of the human RNase $\mathrm{H} 1$ in the pharmacology of DNA-like antisense drugs. J. Biol. Chem. 279, 17181-17189 (2004).

20. Crooke, S. T. Molecular mechanisms of antisense oligonucleotides. Nucleic Acid. Ther. 27, 70-77 (2017).

21. Monia, B. P. et al. Evaluation of 2'-modified oligonucleotides containing 2'-deoxy gaps as antisense inhibitors of gene expression. J. Biol. Chem. 268, 14514-14522 (1993).

22. Liang, X.-H., Sun, H., Nichols, J. G. \& Crooke, S. T. RNase $\mathrm{H} 1$-dependent antisense oligonucleotides are robustly active in directing RNA cleavage in both the cytoplasm and the nucleus. Mol. Ther. $\mathbf{2 5}$ 2075-2092 (2017).

23. Lennox, K. A. \& Behlke, M. A. Cellular localization of long non-coding RNAs affects silencing by RNAi more than by antisense oligonucleotides. Nucleic Acids Res. 44, 863-877 (2016).

24. Dominski, Z. \& Kole, R. Restoration of correct splicing in thalassemic pre-mRNA by antisense oligonucleotides. Proc. Natl Acad. Sci. USA 90, 8673-8677 (1993).

25. Singh, R. N. \& Singh, N. N. Mechanism of splicing regulation of spinal muscular atrophy genes. Adv. Neurobiol. 20, 31-61 (2018).

26. Aartsma-Rus, A. et al. Development of exon skipping therapies for Duchenne muscular dystrophy: a critical review and a perspective on the outstanding issues. Nucleic Acid. Ther. 27, 251-259 (2017).
27. Wan, L. \& Dreyfuss, G. Splicing-correcting therapy for SMA. Cell 170, 5 (2017).

28. Ward, A. J., Norrbom, M., Chun, S., Bennett, C. F. $\&$ Rigo, F. Nonsense-mediated decay as a terminating mechanism for antisense oligonucleotides. Nucleic Acids Res. 42, 5871-5879 (2014).

29. Boiziau, C. et al. Inhibition of translation initiation by antisense oligonucleotides via an RNase-H independent mechanism. Nucleic Acids Res. 19, 1113-1119 (1991)

30. Baker, B. F. et al. 2'-O-(2-methoxy)ethyl-modified anti-intercellular adhesion molecule 1 (ICAM-1) oligonucleotides selectively increase the ICAM-1 mRNA level and inhibit formation of the ICAM-1 translation initiation complex in human umbilical vein endothelial cells. J. Biol. Chem. 272, 11994-12000 (1997).

31. Calvo, S. E., Pagliarini, D. J. \& Mootha, V. K. Upstream open reading frames cause widespread reduction of protein expression and are polymorphic among humans. Proc. Natl Acad. Sci. USA 106 7507-7512 (2009).

32. Liang, X.-H. et al. Translation efficiency of mRNAs is increased by antisense oligonucleotides targeting upstream open reading frames. Nat. Biotechnol. 34 875-880 (2016).

33. Nomakuchi, T. T., Rigo, F., Aznarez, I. \& Krainer, A. R. Antisense oligonucleotide-directed inhibition of nonsense-mediated mRNA decay. Nat. Biotechnol. 34, 164-166 (2016)

34. Vickers, T. A., Wyatt, J. R., Burckin, T., Bennett, C. F. \& Freier, S. M. Fully modified 2' MOE oligonucleotides redirect polyadenylation. Nucleic Acids Res. 29 1293-1299 (2001).

35. Elbashir, S. M. et al. Duplexes of 21-nucleotide RNAs mediate RNA interference in cultured mammalian cells. Nature 411, 494-498 (2001)

36. Liu, J. et al. Argonaute 2 is the catalytic engine of mammalian RNAi. Science 305, 1437-1441 (2004).

37. Roberts, T. C. The microRNA machinery. Adv. Exp. Med. Biol. 887, 15-30 (2015)

38. Schürmann, N., Trabuco, L. G., Bender, C., Russell, R. B. $\&$ Grimm, D. Molecular dissection of human Argonaute proteins by DNA shuffling. Nat. Struct. Mol. Biol. 20 818-826 (2013)

39. Kim, D.-H. et al. Synthetic dsRNA Dicer substrates enhance RNAi potency and efficacy. Nat. Biotechnol. 23, 222-226 (2005)

40. Bramsen, J. B. et al. Improved silencing properties using small internally segmented interfering RNAs. Nucleic Acids Res. 35, 5886-5897 (2007).

41. Byrne, M. et al. Novel hydrophobically modified asymmetric RNAi compounds (sd-rxRNA) demonstrate robust efficacy in the eye. J. Ocul. Pharmacol. Ther. 29, 855-864 (2013).

42. Yu, D. et al. Single-stranded RNAs use RNAi to potently and allele-selectively inhibit mutant huntingtin expression. Cell 150, 895-908 (2012).

43. Lima, W. F. et al. Single-stranded siRNAs activate RNAi in animals. Cell 150, 883-894 (2012).

44. Alterman, J. F. et al. A divalent siRNA chemical scaffold for potent and sustained modulation of gene expression throughout the central nervous system. Nat. Biotechnol. 37, 884-894 (2019).

45. Svoronos, A. A., Engelman, D. M. \& Slack, F. J. OncomiR or tumor suppressor? The duplicity of microRNAs in cancer. Cancer Res. 76, 3666-3670 (2016).

46. Okada, N. et al. A positive feedback between p53 and miR-34 miRNAs mediates tumor suppression. Genes Dev. 28, 438-450 (2014)

47. Bueno, M. J. \& Malumbres, M. MicroRNAs and the cell cycle. Biochim. Biophys. Acta 1812, 592-601 (2011).

48. Shimakami, T. et al. Stabilization of hepatitis $C$ virus RNA by an Ago2-miR-122 complex. Proc. Natl Acad Sci. USA 109, 941-946 (2012).

49. Balasubramaniam, M., Pandhare, J. \& Dash, C. Are microRNAs important players in HIV-1 infection? An update. Viruses 10, 110 (2018)

50. Xu, S. J., Hu, H. T., Li, H. L. \& Chang, S. The role of miRNAs in immune cell development, immune cell activation, and tumor immunity: with a focus on macrophages and natural killer cells. Cells 8, 1140 (2019).

51. Wendt, A., Esguerra, J. L. \& Eliasson, L. Islet microRNAs in health and type-2 diabetes. Curr. Opin. Pharmacol. 43, 46-52 (2018).

52. Vienberg, S., Geiger, J., Madsen, S. \& Dalgaard, L. T. MicroRNAs in metabolism. Acta Physiol. 219 346-361 (2017).

53. van Rooij, E., Liu, N. \& Olson, E. N. MicroRNAs flex their muscles. Trends Genet. 24, 159-166 (2008).
54. Chen, J.-F. et al. microRNA-1 and microRNA-206 regulate skeletal muscle satellite cell proliferation and differentiation by repressing Pax7. J. Cell Biol. 190 867-879 (2010)

55. Roberts, T. C. et al. Expression analysis in multiple muscle groups and serum reveals complexity in the microRNA transcriptome of the MDX mouse with implications for therapy. Mol. Ther. Nucleic Acids 1 , e39 (2012)

56. Cacchiarelli, D. et al. MicroRNAs involved in molecular circuitries relevant for the Duchenne muscular dystrophy pathogenesis are controlled by the dystrophin/nNOS pathway. Cell Metab. 12, 341-351 (2010).

57. Provost, P. et al. Ribonuclease activity and RNA binding of recombinant human Dicer. EMBO J. 21, 5864-5874 (2002).

58. Lee, Y. et al. The nuclear RNase III Drosha initiates microRNA processing. Nature 425, 415-419 (2003).

59. Guo, H., Ingolia, N. T., Weissman, J. S. \& Bartel, D. P. Mammalian microRNAs predominantly act to decrease target mRNA levels. Nature 466, 835-840 (2010).

60. Filipowicz, W., Bhattacharyya, S. N. \& Sonenberg, N. Mechanisms of post-transcriptional regulation by microRNAs: are the answers in sight? Nat. Rev. Genet. 9, 102-114 (2008).

61. Roberts, T. C \& Wood, M J. A. Therapeutic targeting of non-coding RNAs. Essays Biochem. 54, 127-145 (2013).

62. Krützfeldt, J. et al. Silencing of microRNAs in vivo with antagomirs'. Nature 438, 685-689 (2005).

63. Lindow, M. \& Kauppinen, S. Discovering the first microRNA-targeted drug. J. Cell Biol. 199, 407-412 (2012).

64. Jopling, C. L., Yi, M., Lancaster, A. M., Lemon, S. M $\&$ Sarnow, P. Modulation of hepatitis C virus RNA abundance by a liver-specific microRNA. Science 309 1577-1581 (2005)

65. Jopling, C. L., Schütz, S. \& Sarnow, P. Position-dependent function for a tandem microRNA miR-122-binding site located in the hepatitis $\mathrm{C}$ virus RNA genome. Cell Host Microbe 4, 77-85 (2008).

66. Jopling, C. L. Targeting microRNA-122 to treat hepatitis $C$ virus infection. Viruses 2, 1382-1393 (2010).

67. Janssen, H. L. A. et al. Treatment of HCV infection by targeting microRNA. N. Engl. J. Med. 368, 1685-1694 (2013).

68. Machlin, E. S., Sarnow, P. \& Sagan, S. M. Masking the $5^{\prime}$ terminal nucleotides of the hepatitis $C$ virus genome by an unconventional microRNA-target RNA complex. Proc. Natl Acad. Sci. USA 108, 3193-3198 (2011).

69. Ottosen, S. et al. In vitro antiviral activity and preclinical and clinical resistance profile of miravirsen, a novel anti-hepatitis $C$ virus therapeutic targeting the human factor miR-122. Antimicrob. Agents Chemother 59, 599-608 (2015).

70. Gomez, I. G. et al. Anti-microRNA-21 oligonucleotides prevent Alport nephropathy progression by stimulating metabolic pathways. J. Clin. Invest. 125, 141-156 (2015).

71. Lee, E. C. et al. Discovery and preclinical evaluation of anti-miR-17 oligonucleotide RGLS4326 for the treatment of polycystic kidney disease. Nat. Commun. 10, 1-14 (2019)

72. Seto, A. G. et al. Cobomarsen, an oligonucleotide inhibitor of miR-155, co-ordinately regulates multiple survival pathways to reduce cellular proliferation and survival in cutaneous T-cell lymphoma. Br. J. Haematol. 183, 428-444 (2018)

73. Gallant-Behm, C. L. et al. A microRNA-29 mimic (remlarsen) represses extracellular matrix expression and fibroplasia in the skin. J. Invest. Dermatol. 139, 1073-1081 (2019).

74. Choi, W.-Y., Giraldez, A. J. \& Schier, A. F. Target protectors reveal dampening and balancing of Nodal agonist and antagonist by miR-430. Science 318 , 271-274 (2007).

75. Wang, Z. The principles of miRNA-masking antisense oligonucleotides technology. Methods Mol. Biol. 676 43-49 (2011)

76. Roberts, T. C., Morris, K. V. \& Wood, M. J. A. The role of long non-coding RNAs in neurodevelopment, brain function and neurological disease. Philos. Trans. $R$. Soc. Lond. B Biol. Sci. 369, (2014).

77. Wahlestedt, C. Targeting long non-coding RNA to therapeutically upregulate gene expression. Nat. Rev. Drug Discov. 12, 433-446 (2013).

78. Yoon, S. \& Rossi, J. J. Therapeutic potential of small activating RNAs (saRNAs) in human cancers. Curr. Pharm. Biotechnol. 19, 604-610 (2018). 
79. Faghihi, M. A. et al. Expression of a noncoding RNA is elevated in Alzheimer's disease and drives rapid feed-forward regulation of $\beta$-secretase. Nat. Med. 14, 723-730 (2008)

80. Modarresi, F. et al. Inhibition of natural antisense transcripts in vivo results in gene-specific transcriptional upregulation. Nat. Biotechnol. 30 453-459 (2012)

81. Meng, L. et al. Towards a therapy for Angelman syndrome by targeting a long non-coding RNA. Nature 518, 409-412 (2015).

82. Hsiao, J. et al. Upregulation of haploinsufficient gene expression in the brain by targeting a long non-coding RNA improves seizure phenotype in a model of Dravet syndrome. EBioMedicine 9, 257-277 (2016).

83. Schwartz, J. C. et al. Antisense transcripts are targets for activating small RNAs. Nat. Struct. Mol. Biol. 15 , 842-848 (2008)

84. Matsui, M. et al. Promoter RNA links transcriptional regulation of inflammatory pathway genes. Nucleic Acids Res. 41, 10086-10109 (2013).

85. Turunen, T. A. et al. Changes in nuclear and cytoplasmic microRNA distribution in response to hypoxic stress. Sci. Rep. 9, 10332 (2019).

86. Gagnon, K. T., Li, L., Chu, Y., Janowski, B. A. \& Corey, D. R. RNAi factors are present and active in human cell nuclei. Cell Rep. 6, 211-221 (2014).

87. Voutila, J. et al. Development and mechanism of small activating RNA targeting CEBPA, a novel therapeutic in clinical trials for liver cancer. Mol. Ther. $\mathbf{2 5}$ 2705-2714 (2017)

88. Reebye, V. et al. Gene activation of CEBPA using saRNA: preclinical studies of the first in human saRNA drug candidate for liver cancer. Oncogene 37 3216-3228 (2018)

89. Sarker, D. et al. MTL-CEBPA, a small activating RNA therapeutic up-regulating C/EBP- $\alpha$, in patients with advanced liver cancer: a first-in-human, multi-centre, open-label, phase I trial. Clin. Cancer Res. https:// doi.org/10.1158/1078-0432.CCR-20-0414 (2020). This paper presents the currently most advanced oligonucleotide therapy aimed at upregulating a target gene.

90. Tsui, N. B. Y., Ng, E. K. O. \& Lo, Y. M. D. Stability of endogenous and added RNA in blood specimens, serum, and plasma. Clin. Chem. 48, 1647-1653 (2002)

91. Iversen, F. et al. Optimized siRNA-PEG conjugates for extended blood circulation and reduced urine excretion in mice. Theranostics 3, 201-209 (2013)

92. Geary, R. S., Norris, D., Yu, R. \& Bennett, C. F. Pharmacokinetics, biodistribution and cell uptake of antisense oligonucleotides. Adv. Drug Deliv. Rev. 87 46-51 (2015).

93. Shemesh, C. S. et al. Pharmacokinetic and pharmacodynamic investigations of ION-353382, a model antisense oligonucleotide: using $\alpha_{2}$ macroglobulin and murinoglobulin double-knockout mice. Nucleic Acid. Ther. 26, 223-235 (2016).

94. Allen, T. M. The use of glycolipids and hydrophilic polymers in avoiding rapid uptake of liposomes by the mononuclear phagocyte system. Adv. Drug Delivery Rev. 13, 285-309 (1994).

95. Sahay, G. et al. Efficiency of siRNA delivery by lipid nanoparticles is limited by endocytic recycling. Nat. Biotechnol. 31, 653-658 (2013).

96. Finkel, R. S. et al. Treatment of infantile-onset spinal muscular atrophy with nusinersen: a phase 2 , open-label, dose-escalation study. Lancet 388, 3017-3026 (2016). This landmark study shows efficacy of an oligonucleotide therapy for spinal muscular atrophy.

97. Wan, W. B. \& Seth, P. P. The medicinal chemistry of therapeutic oligonucleotides. J. Med. Chem. 59, 9645-9667 (2016).

98. Hung, G. et al. Characterization of target mRNA reduction through in situ RNA hybridization in multiple organ systems following systemic antisense treatment in animals. Nucleic Acid. Ther. 23, 369-378 (2013).

99. Tabrizi, S. J. et al. Targeting huntingtin expression in patients with Huntington's disease. N. Engl. J. Med. 380, 2307-2316 (2019).

100. Eckstein, F. Phosphorothioates, essential components of therapeutic oligonucleotides. Nucleic Acid. Ther. 24, 374-387 (2014).

101. Hall, A. H. S., Wan, J., Shaughnessy, E. E., Ramsay Shaw, B. \& Alexander, K. A. RNA interference using boranophosphate siRNAs: structure-activity relationships. Nucleic Acids Res. 32, 5991-6000 (2004).

102. Braasch, D. A. et al. RNA interference in mammalian cells by chemically-modified RNA. Biochemistry 42, 7967-7975 (2003).
103. Bijsterbosch, M. K. et al. In vivo fate of phosphorothioate antisense oligodeoxynucleotides: predominant uptake by scavenger receptors on endothelial liver cells. Nucleic Acids Res. 25 , 3290-3296 (1997).

104. Ezzat, K. et al. Self-assembly into nanoparticles is essential for receptor mediated uptake of therapeutic antisense oligonucleotides. Nano Lett. 15, 4364-4373 (2015).

105. Miller, C. M. et al. Stabilin-1 and Stabilin-2 are specific receptors for the cellular internalization of phosphorothioate-modified antisense oligonucleotides (ASOs) in the liver. Nucleic Acids Res. 44, 2782-2794 (2016).

106. Gaus, H. J. et al. Characterization of the interactions of chemically-modified therapeutic nucleic acids with plasma proteins using a fluorescence polarization assay. Nucleic Acids Res. 47, 1110-1122 (2019).

107. Brown, D. A. et al. Effect of phosphorothioate modification of oligodeoxynucleotides on specific protein binding. J. Biol. Chem. 269, 26801-26805 (1994).

108. Liang, X., Sun, H., Shen, W. \& Crooke, S. T Identification and characterization of intracellular proteins that bind oligonucleotides with phosphorothioate linkages. Nucleic Acids Res. 43 2927-2945 (2015).

109. Weidner, D. A., Valdez, B. C., Henning, D., Greenberg, S $\&$ Busch, H. Phosphorothioate oligonucleotides bind in a non sequence-specific manner to the nucleolar protein C23/nucleolin. FEBS Lett. 366, 146-150 (1995).

110. Shen, W., Liang, X. \& Crooke, S. T. Phosphorothioate oligonucleotides can displace NEAT1 RNA and form nuclear paraspeckle-like structures. Nucleic Acids Res. 42, 8648-8662 (2014)

111. Liang, X., Shen, W., Sun, H., Prakash, T. P. \& Crooke, S. T. TCP1 complex proteins interact with phosphorothioate oligonucleotides and can co-localize in oligonucleotide-induced nuclear bodies in mammalian cells. Nucleic Acids Res. 42, 7819-7832 (2014).

112. Monia, B. P., Johnston, J. F., Sasmor, H. \& Cummins, L. L. Nuclease resistance and antisense activity of modified oligonucleotides targeted to Ha-ras. J. Biol. Chem. 271 14533-14540 (1996).

113. Iwamoto, N. et al. Control of phosphorothioate stereochemistry substantially increases the efficacy of antisense oligonucleotides. Nat. Biotechnol. 35, 845-851 (2017).

This study demonstrates the influence of phosphorothioate stereochemistry on the properties of gapmer ASOs.

114. Wexler, M. M. S. Wave Life Sciences discontinues development of suvodirsen for DMD. Muscular Dystrophy News https://musculardystrophynews.com/ 2019/12/17/wave-life-sciences-discontinuessuvodirsen-development-for-dmd/ (2019).

115. Wan, W. B. et al. Synthesis, biophysical properties and biological activity of second generation antisense oligonucleotides containing chiral phosphorothioate linkages. Nucleic Acids Res. 42, 13456-13468 (2014).

116. Lee, H.-S. et al. Abasic pivot substitution harnesses target specificity of RNA interference. Nat. Commun 6, 10154 (2015)

117. Liu, J. et al. RNA duplexes with abasic substitutions are potent and allele-selective inhibitors of huntingtin and ataxin-3 expression. Nucleic Acids Res. 41 8788-8801 (2013)

118. Schirle, N. T. \& MacRae, I. J. The crystal structure of human Argonaute2. Science 336, 1037-1040 (2012).

119. Frank, F., Sonenberg, N. \& Nagar, B. Structural basis for 5 '-nucleotide base-specific recognition of guide RNA by human AGO2. Nature 465, 818-822 (2010)

120. Haraszti, R. A. et al. 5'-Vinylphosphonate improves tissue accumulation and efficacy of conjugated siRNAs in vivo. Nucleic Acids Res. 45, 7581-7592 (2017).

121. Behlke, M. A. Chemical modification of siRNAs for in vivo use. Oligonucleotides 18, 305-319 (2008).

122. Southwell, A. L., Skotte, N. H., Bennett, C. F. \& Hayden, M. R. Antisense oligonucleotide therapeutics for inherited neurodegenerative diseases. Trends $\mathrm{Mol}$. Med. 18, 634-643 (2012)

123. Manoharan, M. 2'-Carbohydrate modifications in antisense oligonucleotide therapy: importance of conformation, configuration and conjugation. Biochim. Biophys. Acta 1489, 117-130 (1999).

124. Roberts, T. C., Ezzat, K., El Andaloussi, S. \& Weinberg, M. S. Synthetic siRNA delivery: progress and prospects. Methods Mol. Biol. 1364, 291-310 (2016).
125. Prakash, T. P. et al. Positional effect of chemical modifications on short interference RNA activity in mammalian cells. J. Med. Chem. 48, 4247-4253 (2005).

126. Allerson, C. R. et al. Fully 2'-modified oligonucleotide duplexes with improved in vitro potency and stability compared to unmodified small interfering RNA. J. Med. Chem. 48, 901-904 (2005).

127. Hassler, M. R. et al. Comparison of partially and fully chemically-modified siRNA in conjugate-mediated delivery in vivo. Nucleic Acids Res. 46, 2185-2196 (2018).

128. Jackson, A. L. et al. Position-specific chemical modification of siRNAs reduces 'off-target' transcript silencing. RNA 12, 1197-1205 (2006).

129. Springer, A. D. \& Dowdy, S. F. GalNAc-siRNA conjugates: leading the way for delivery of RNAi therapeutics. Nucleic Acid. Ther. 28, 109-118 (2018).

130. Garber, K. Alnylam terminates revusiran program, stock plunges. Nat. Biotechnol. 34, 1213-1214 (2016).

131. Nair, J. K. et al. Impact of enhanced metabolic stability on pharmacokinetics and pharmacodynamics of GalNAc-siRNA conjugates. Nucleic Acids Res. 45, 10969-10977 (2017).

132. Judge, A. D. et al. Sequence-dependent stimulation of the mammalian innate immune response by synthetic siRNA. Nat. Biotechnol, 23, 457-462 (2005).

133. Shen, W., Liang, X.-H., Sun, H. \& Crooke, S. T. 2'-Fluoro-modified phosphorothioate oligonucleotide can cause rapid degradation of P54nrb and PSF, Nucleic Acids Res. 43, 4569-4578 (2015).

134. Iwasaki, A. \& Medzhitov, R. Toll-like receptor control of the adaptive immune responses. Nat. Immunol. 5, 987-995 (2004).

135. Hornung, V. et al. Sequence-specific potent induction of IFN- $\alpha$ by short interfering RNA in plasmacytoid dendritic cells through TLR7. Nat. Med. 11, 263-270 (2005).

136. Hartmann, G. Nucleic acid immunity. Adv. Immunol. 133, 121-169 (2017).

137. Morrissey, D. V. et al. Potent and persistent in vivo anti-HBV activity of chemically modified siRNAs. Nat. Biotechnol. 23, 1002-1007 (2005). This landmark study demonstrates enhanced RNAi-mediated gene silencing using chemically modified SiRNA in vivo.

138. Judge, A. D., Bola, G., Lee, A. C. H. \& MacLachlan, I. Design of noninflammatory synthetic siRNA mediating potent gene silencing in vivo. Mol. Ther. 13, 494-505 (2006)

139. Poeck, H. et al. 5'-Triphosphate-siRNA: turning gene silencing and Rig-l activation against melanoma. Nat. Med. 14, 1256-1263 (2008).

140. Kortylewski, M. et al. In vivo delivery of siRNA to immune cells by conjugation to a TLR9 agonist enhances antitumor immune responses. Nat. Biotechnol. 27, 925-932 (2009).

141. Kanzler, H., Barrat, F. J., Hessel, E. M. \& Coffman, R. L. Therapeutic targeting of innate immunity with Toll-like receptor agonists and antagonists. Nat. Med. 13, 552-559 (2007)

142. Kandimalla, E. R. et al. Design, synthesis and biological evaluation of novel antagonist compounds of Toll-like receptors 7, 8 and 9. Nucleic Acids Res. 41, 3947-3961 (2013).

143. Veedu, R. N. \& Wengel, J. Locked nucleic acid as a novel class of therapeutic agents. RNA Biol. 6 , 321-323 (2009).

144. Vester, B. \& Wengel, J. LNA (locked nucleic acid): high-affinity targeting of complementary RNA and DNA. Biochemistry 43, 13233-13241 (2004).

145. Morita, K. et al. 2'-O,4'-C-ethylene-bridged nucleic acids (ENA): highly nuclease-resistant and thermodynamically stable oligonucleotides for antisense drug. Bioorg. Med. Chem. Lett. 12, 73-76 (2002).

146. Hong, D. et al AZD9150, a next-generation antisense oligonucleotide inhibitor of STAT3 with early evidence of clinical activity in lymphoma and lung cancer. Sci. Transl Med. 7, 314 ra185 (2015)

147. Koshkin, A. A. et al. LNA (locked nucleic acids): synthesis of the adenine, cytosine, guanine, 5-methylcytosine, thymine and uracil bicyclonucleoside monomers, oligomerisation, and unprecedented nucleic acid recognition. Tetrahedron 54, 3607-3630 (1998).

148. Obad, S. et al. Silencing of microRNA families by seed-targeting tiny LNAs. Nat. Genet. 43, 371-378 (2011).

149. Moulton, J. D. Using morpholinos to control gene expression. Curr. Protoc. Nucleic Acid. Chem. 68 4.30.1-4.30.29 (2017). 
150. Iversen, P. L. Phosphorodiamidate morpholino oligomers: favorable properties for sequence-specific gene inactivation. Curr. Opin. Mol. Ther. 3, 235-238 (2001).

151. Komaki, H. et al. Systemic administration of the antisense oligonucleotide NS-065/NCNP-01 for skipping of exon 53 in patients with Duchenne muscular dystrophy. Sci. Transl Med. 10, eaan0713 (2018).

152. Larsen, H. J., Bentin, T. \& Nielsen, P. E. Antisense properties of peptide nucleic acid. Biochim. Biophys. Acta 1489, 159-166 (1999).

153. Saarbach, J., Sabale, P. M. \& Winssinger, N. Peptide nucleic acid (PNA) and its applications in chemical biology, diagnostics, and therapeutics. Curr. Opin. Chem. Biol. 52, 112-124 (2019).

154. Renneberg, D. \& Leumann, C. J. Watson-Crick base-pairing properties of tricyclo-DNA. J. Am. Chem. Soc. 124, 5993-6002 (2002)

155. Goyenvalle, A. et al. Functional correction in mouse models of muscular dystrophy using exon-skipping tricyclo-DNA oligomers. Nat. Med. 21, 270-275 (2015).

156. Dowling, J. J. Eteplirsen therapy for Duchenne muscular dystrophy: skipping to the front of the line. Nat. Rev. Neurol. 12, 675-676 (2016).

157. Yin, H. et al. Optimization of peptide nucleic acid antisense oligonucleotides for local and systemic dystrophin splice correction in the MDX mouse. Mol. Ther. 18, 819-827 (2010).

158. Imbert, M., Blandel, F., Leumann, C., Garcia, L. $\Sigma$ Goyenvalle, A. Lowering mutant huntingtin using tricyclo-DNA antisense oligonucleotides as a therapeutic approach for Huntington's disease. Nucleic Acid. Ther. 29, 256-265 (2019).

159. Meade, B. R. et al. Efficient delivery of RNAi prodrugs containing reversible charge-neutralizing phosphotriester backbone modifications. Nat. Biotechnol. 32 , 1256-1261 (2014)

160. Wolfrum, C. et al. Mechanisms and optimization of in vivo delivery of lipophilic siRNAs. Nat. Biotechnol. 25, 1149-1157 (2007).

161. Soutschek, J. et al. Therapeutic silencing of an endogenous gene by systemic administration of modified siRNAs. Nature 432, 173-178 (2004).

162. Lorenz, C., Hadwiger, P., John, M., Vornlocher, H.-P. \& Unverzagt, C. Steroid and lipid conjugates of siRNAs to enhance cellular uptake and gene silencing in liver cells. Bioorg. Med. Chem. Lett. 14, 4975-4977 (2004).

163. Eguchi, A. et al. Efficient siRNA delivery into primary cells by a peptide transduction domain-dsRNA binding domain fusion protein. Nat. Biotechnol. 27 567-571 (2009)

164. Betts, C. et al. Pip6-PMO, a new generation of peptide-oligonucleotide conjugates with improved cardiac exon skipping activity for DMD treatment. Mol. Ther. Nucleic Acids 1, e38 (2012).

165. Alam, Md. R. et al. Multivalent cyclic RGD conjugates for targeted delivery of small interfering RNA Bioconjugate Chem. 22, 1673-1681 (2011)

166. Ämmälä, C. et al. Targeted deliivery of antisense oligonucleotides to pancreatic $\beta$-cells. Sci. Adv. 4 eaat3386 (2018)

167. Liu, X. et al. Tumor-targeted in vivo gene silencing via systemic delivery of CRGD-conjugated siRNA. Nucleic Acids Res. 42, 11805-11817 (2014).

168. McNamara, J. O et al. Cell type-specific delivery of siRNAs with aptamer-siRNA chimeras. Nat. Biotechnol. 24, 1005-1015 (2006)

169. Song, E. et al. Antibody mediated in vivo delivery of small interfering RNAs via cell-surface receptors. Nat. Biotechnol. 23, 709-717 (2005). This paper demonstrates antibody-siRNA conjugates for gene silencing in mice.

170. Nair, J. K. et al. Multivalent $N$-acetylgalactosamineconjugated siRNA localizes in hepatocytes and elicits robust RNAi-mediated gene silencing. J. Am. Chem. Soc 136, 16958-16961 (2014). This paper demonstrates long-term gene silencing after weekly dosing of an optimized GalNAc-siRNA conjugate in mice.

171. Matsuda, S. et al. siRNA conjugates carrying sequentially assembled trivalent $N$-acetylgalactosamine linked through nucleosides elicit robust gene silencing in vivo in hepatocytes. ACS Chem. Biol. 10, 1181-1187 (2015).

172. Tai, W. Current aspects of siRNA bioconjugate for in vitro and in vivo delivery. Molecules 24, 2211 (2019)

173. Juliano, R. L. The delivery of therapeutic oligonucleotides. Nucleic Acids Res. 44, 6518-6548 (2016).
174. Khan, T. et al. Silencing myostatin using cholesterolconjugated siRNAs induces muscle growth. Mol. Ther Nucleic Acids 5, e342 (2016).

175. Nishina, K. et al. Efficient in vivo delivery of siRNA to the liver by conjugation of $\alpha$-tocopherol. Mol. Ther. 16 734-740 (2008).

176. Osborn, M. F et al. Hydrophobicity drives the systemic distribution of lipid-conjugated siRNAs via lipid transport pathways. Nucleic Acids Res. 47 1070-1081 (2019).

177. Spiess, M. The asialoglycoprotein receptor: a mode for endocytic transport receptors. Biochemistry 29, 10009-10018 (1990).

178. Tanowitz, M. et al. Asialoglycoprotein receptor 1 mediates productive uptake of $\mathrm{N}$-acetylgalactosamineconjugated and unconjugated phosphorothioate antisense oligonucleotides into liver hepatocytes. Nucleic Acids Res. 45, 12388-12400 (2017).

179. Prakash, T. P. et al. Targeted delivery of antisense oligonucleotides to hepatocytes using triantennary $\mathrm{N}$-acetyl galactosamine improves potency 10 -fold in mice. Nucleic Acids Res. 42, 8796-8807 (2014).

180. Ray, K. K. et al. Inclisiran in patients at high cardiovascular risk with elevated LDL cholesterol. N. Engl. J. Med. 376, 1430-1440 (2017).

181. Zimmermann, T. S. et al. Clinical proof of concept for a novel hepatocyte-targeting GalNAc-siRNA conjugate. Mol. Ther. 25, 71-78 (2017).

182. Viney, N. J. et al. Antisense oligonucleotides targeting apolipoprotein(a) in people with raised lipoprotein(a): two randomised, double-blind, placebo-controlled, dose-ranging trials. Lancet 388, 2239-2253 (2016).

183. Sardh, E. et al. Phase 1 trial of an RNA interference therapy for acute intermittent porphyria. N. Engl. J. Med. 380, 549-558 (2019)

184. No authors listed. Novartis to acquire the medicines company for USD 9.7 bn, adding inclisiran, a potentially transformational investigational cholesterol-lowering therapy to address leading global cause of death. Novartis https://www.novartis.com/ news/media-releases/novartis-acquire-medicinescompany-usd-97-bn-adding-inclisiran-potentiallytransformational-investigational-cholesterol-loweringtherapy-address-leading-global (2019).

185. Hooper, A. J. \& Burnett, J. R. Anti-PCSK9 therapies for the treatment of hypercholesterolemia. Expert Opin. Biol. Ther. 13, 429-435 (2013).

186. Mousavi, S. A., Berge, K. E. \& Leren, T. P. The unique role of proprotein convertase subtilisin/kexin 9 in cholesterol homeostasis. J. Intern. Med. 266 507-519 (2009).

187. Fitzgerald, K. et al. A highly durable RNAi therapeutic inhibitor of PCSK9. N. Engl. J. Med. 376, 41-51 (2017).

188. Sievers, E. L. \& Senter, P. D. Antibody-drug conjugates in cancer therapy. Annu. Rev. Med. 64, 15-29 (2013).

189. Yao, Y. et al. Targeted delivery of PLK1-siRNA by ScFv suppresses Her2+ breast cancer growth and metastasis. Sci. Transl Med. 4, 130 ra48 (2012).

190. Kumar, P. et al. T cell-specific siRNA delivery suppresses HIV-1 infection in humanized mice. Cell 134, 577-586 (2008).

191. Sugo, T. et al. Development of antibody-siRNA conjugate targeted to cardiac and skeletal muscles. J. Control. Rel. 237, 1-13 (2016).

192. Cuellar, T. L. et al. Systematic evaluation of antibody-mediated siRNA delivery using an industrial platform of THIOMAB-siRNA conjugates. Nucleic Acids Res. 43, 1189-1203 (2015).

193. Arnold, A. E. et al. Antibody-antisense oligonucleotide conjugate downregulates a key gene in glioblastoma stem cells. Mol. Ther. Nucleic Acids 11, 518-527 (2018).

194. Astriab-Fisher, A., Fisher, M. H., Juliano, R. \& Herdewijn, P. Increased uptake of antisense oligonucleotides by delivery as double stranded complexes. Biochem. Pharmacol. 68, 403-407 (2004).

195. Nuzzo, S. et al. Potential and challenges of aptamers as specific carriers of therapeutic oligonucleotides for precision medicine in cancer. Cancers 11, 1521 (2019).

196. Hong, S., Sun, N., Liu, M., Wang, J. \& Pei, R. Building a chimera of aptamer-antisense oligonucleotide for silencing galectin-1 gene. RSC Adv. 6, 112445-112450 (2016).

197. White, R. R., Sullenger, B. A. \& Rusconi, C. P. Developing aptamers into therapeutics. J. Clin. Invest. 106 929-934 (2000)

198. McClorey, G. \& Banerjee, S. Cell-penetrating peptides to enhance delivery of oligonucleotide-based therapeutics. Biomedicines 6, 51 (2018).
199. Yin H. et al. Cell-penetrating peptide-conjugated antisense oligonucleotides restore systemic muscle and cardiac dystrophin expression and function. Hum. Mol. Genet. 17, 3909-3918 (2008).

200. McClorey, G., Moulton, H. M., Iversen, P. L., Fletcher, S. $\&$ Wilton, S. D. Antisense oligonucleotide-induced exon skipping restores dystrophin expression in vitro in a canine model of DMD. Gene Ther. 13, 1373-1381 (2006).

201. Wu, B. et al. Effective rescue of dystrophin improves cardiac function in dystrophin-deficient mice by a modified morpholino oligomer. Proc. Natl Acad. Sci. USA 105, 14814-14819 (2008)

202. Abes, R. et al. Delivery of steric block morpholino oligomers by $(\mathrm{R}-\mathrm{X}-\mathrm{R})_{4}$ peptides: structure-activity studies. Nucleic Acids Res. 36, 6343-6354 (2008)

203. Wender, P. A. et al. The design, synthesis, and evaluation of molecules that enable or enhance cellular uptake: peptoid molecular transporters. Proc. Natl Acad. Sci. USA 97, 13003-13008 (2000).

204. Yin, H. et al. A fusion peptide directs enhanced systemic dystrophin exon skipping and functional restoration in dystrophin-deficient mdx mice. Hum. Mol. Genet. 18, 4405-4414 (2009).

205. Yin, H. et al. Context dependent effects of chimeric peptide morpholino conjugates contribute to dystrophin exon-skipping efficiency. Mol. Ther. Nucleic Acids $\mathbf{2}$, e124 (2013)

206. Gao, X. et al. Effective dystrophin restoration by a novel muscle-homing peptide-morpholino conjugate in dystrophin-deficient MDX mice. Mol. Ther. 22 1333-1341 (2014).

207. Yin, H. et al. Pip5 transduction peptides direct high efficiency oligonucleotide-mediated dystrophin exon skipping in heart and phenotypic correction in MDX mice. Mol. Ther. 19, 1295-1303 (2011).

208. van Westering, T. L. E. et al. Uniform sarcolemmal dystrophin expression is required to prevent extracellular microRNA release and improve dystrophic pathology. J. Cachexia Sarcopenia Muscle 11, 578-593 (2019).

209. Gait, M. J. et al. Cell-penetrating peptide conjugates of steric blocking oligonucleotides as therapeutics for neuromuscular diseases from a historical perspective to current prospects of treatment. Nucleic Acid. Ther 29, 1-12 (2019).

210. Betts, C. A. et al. Prevention of exercised induced cardiomyopathy following Pip-PMO treatment in dystrophic mdx mice. Sci. Rep. 5, 8986 (2015)

211. Hammond, S. M. et al. Systemic peptide-mediated oligonucleotide therapy improves long-term survival in spinal muscular atrophy. Proc. Natl Acad. Sci. USA 113, 10962-10967 (2016)

212. Amantana, A. et al. Pharmacokinetics, biodistribution, stability and toxicity of a cell-penetrating peptidemorpholino oligomer conjugate. Bioconjug. Chem. 18 1325-1331 (2007)

213. Moulton, H. M. \& Moulton, J. D. Morpholinos and their peptide conjugates: therapeutic promise and challenge for Duchenne muscular dystrophy. Biochim. Biophys. Acta 1798, 2296-2303 (2010).

214. Lehto, T. et al. Cellular trafficking determines the exon skipping activity of Pip6a-PMO in MDX skeletal and cardiac muscle cells. Nucleic Acids Res. 42 3207-3217 (2014).

215. Abes, S. et al. Vectorization of morpholino oligomers by the (R-Ahx-R) $)_{4}$ peptide allows efficient splicing correction in the absence of endosomolytic agents. J. Control. Rel. 116, 304-313 (2006).

216. Bestas, B. et al. Splice-correcting oligonucleotides restore BTK function in X-linked agammaglobulinemia model. J. Clin. Invest. 124, 4067-4081 (2014).

217. Du, L. et al. Arginine-rich cell-penetrating peptide dramatically enhances AMO-mediated ATM aberrant splicing correction and enables delivery to brain and cerebellum. Hum. Mol. Genet. 20, 3151-3160 (2011).

218. Geller, B. L. et al. Gene-silencing antisense oligomers inhibit acinetobacter growth in vitro and in vivo. J. Infect. Dis. 208, 1553-1560 (2013).

219. Burrer, R. et al. Antiviral effects of antisense morpholino oligomers in murine coronavirus infection models. J. Virol. 81, 5637-5648 (2007).

220. Neuman, B. W. et al. Inhibition and escape of SARS-CoV treated with antisense morpholino oligomers. Adv. Exp. Med. Biol. 581, 567-571 (2006).

221. Enterlein, S. et al. VP35 knockdown inhibits Ebola virus amplification and protects against lethal infection in mice. Antimicrob. Agents Chemother. 50, 984-993 (2006). 
222. Moschos, S. A. et al. Lung delivery studies using siRNA conjugated to TAT (48-60) and penetratin reveal peptide induced reduction in gene expression and induction of innate immunity. Bioconjug. Chem. 18, 1450-1459 (2007).

223. Cavallaro, G., Sardo, C., Craparo, E. F., Porsio, B. \& Giammona, G. Polymeric nanoparticles for siRNA delivery: production and applications. Int. J. Pharm. 525, 313-333 (2017).

224. Dzmitruk, V. et al. Dendrimers show promise for siRNA and microRNA therapeutics. Pharmaceutics 10, 126 (2018)

225. Mignani, S., Shi, X., Zablocka, M. \& Majoral, J.-P. Dendrimer-enabled therapeutic antisense delivery systems as innovation in medicine. Bioconjug. Chem 30, 1938-1950 (2019)

226. Crombez, L. et al. Targeting cyclin B1 through peptide-based delivery of siRNA prevents tumour growth. Nucleic Acids Res. 37, 4559-4569 (2009).

227. El Andaloussi, S. et al. Design of a peptide-based vector, PepFect6, for efficient delivery of siRNA in cell culture and systemically in vivo. Nucleic Acids Res. 39 3972-3987 (2011)

228. Kumar, P. et al. Transvascular delivery of small interfering RNA to the central nervous system. Nature 448, 39-43 (2007)

This study shows the utility of RVG peptide derivatives for delivering siRNA to the brain after intravenous injection in mice.

229. Montrose, K., Yang, Y., Sun, X., Wiles, S. \& Krissansen, G. W. Xentry, a new class of cell-penetrating peptide uniquely equipped for delivery of drugs. Sci. Rep. 3, 1-7 (2013).

230. Tabaković, A., Kester, M. \& Adair, J. H. Calcium phosphate-based composite nanoparticles in bioimaging and therapeutic delivery applications. Wiley Interdiscip. Rev. Nanomed. Nanobiotechnol. 4 96-112 (2012)

231. Semple, S. C. et al. Rational design of cationic lipids for siRNA delivery. Nat. Biotechnol. 28, 172-176 (2010).

232. de Fougerolles, A. R. Delivery vehicles for small interfering RNA in vivo. Hum. Gene Ther. 19, 125-132 (2008).

233. Ambegia, E. et al. Stabilized plasmid-lipid particles containing PEG-diacylglycerols exhibit extended circulation lifetimes and tumor selective gene expression. Biochim. Biophys. Acta 1669, 155-163 (2005).

234. Tam, Y. Y. C., Chen, S. \& Cullis, P. R. Advances in lipid nanoparticles for siRNA delivery. Pharmaceutics $\mathbf{5}$. 498-507 (2013).

235. Zimmermann, T. S. et al. RNAi-mediated gene silencing in non-human primates. Nature 441, 111-114 (2006)

236. Hoy, S. M. Patisiran: first global approval. Drugs $\mathbf{7 8 ,}$ 1625-1631 (2018)

237. Akinc, A. et al. Targeted delivery of RNAi therapeutics with endogenous and exogenous ligand-based mechanisms. Mol. Ther. 18, 1357-1364 (2010).

238. Akinc, A. et al. A combinatorial library of lipid-like materials for delivery of RNAi therapeutics. Nat. Biotechnol. 26, 561-569 (2008).

239. Akinc, A. et al. Development of lipidoid-siRNA formulations for systemic delivery to the liver. Mol. Ther. 17, 872-879 (2009).

240. Love, K. T. et al. Lipid-like materials for low-dose in vivo gene silencing. Proc. Natl Acad. Sci. USA 107, 1864-1869 (2010)

241. Juliano, R. L. \& Akhtar, S. Liposomes as a drug delivery system for antisense oligonucleotides. Antisense Res. Dev. 2, 165-176 (1992).

242. Wisse, E., Jacobs, F., Topal, B., Frederik, P. \& De Geest, B. The size of endothelial fenestrae in human liver sinusoids: implications for hepatocyte-directed gene transfer. Gene Ther. 15, 1193-1199 (2008).

243. Rungta, R. L. et al. Lipid nanoparticle delivery of siRNA to silence neuronal gene expression in the brain. Mol. Ther. Nucleic Acids 2, e136 (2013).

244. Seth, P. P., Tanowitz, M. \& Bennett, C. F. Selective tissue targeting of synthetic nucleic acid drugs. J. Clin. Invest. 129, 915-925 (2019).

245. Endsley, A. N. \& Ho, R. J. Y. Design and characterization of novel peptide-coated lipid nanoparticles for targeting anti-HIV drug to CD4 expressing cells. AAPS J. 14, 225-235 (2012)

246. Suk, J. S., Xu, O., Kim, N., Hanes, J. \& Ensign, L. M. PEGylation as a strategy for improving nanoparticlebased drug and gene delivery. Adv. Drug Delivery Rev. 99, 28-51 (2016).

247. Li, S.-D. \& Huang, L. Targeted delivery of antisense oligodeoxynucleotide and small interference RNA into lung cancer cells. Mol. Pharm. 3, 579-588 (2006).

248. Tam, Y. Y. C. et al. Small molecule ligands for enhanced intracellular delivery of lipid nanoparticle formulations of siRNA. Nanomedicine 9, 665-674 (2013).

249. Sato, Y. et al. Resolution of liver cirrhosis using vitamin A-coupled liposomes to deliver siRNA against a collagen-specific chaperone. Nat. Biotechnol. 26, 431-442 (2008)

250. Coelho, T. et al. Safety and efficacy of RNAi therapy for transthyretin amyloidosis. N. Engl. J. Med. 369 , 819-829 (2013).

251. Wiklander, O. P. B., Brennan, M. Á., Lötvall, J., Breakefield, X. O. \& El Andaloussi, S. Advances in therapeutic applications of extracellular vesicles. Sci. Transl Med. 11, eaav8521 (2019).

252. Kalluri, R. \& LeBleu, V. S. The biology, function, and biomedical applications of exosomes. Science 367 , eaau6977 (2020).

253. Valadi, H. et al. Exosome-mediated transfer of mRNAs and microRNAs is a novel mechanism of genetic exchange between cells. Nat. Cell Biol. 9, 654-659 (2007).

254. Skog, J. et al. Glioblastoma microvesicles transport RNA and proteins that promote tumour growth and provide diagnostic biomarkers. Nat. Cell Biol. 10 , 1470-1476 (2008)

255. Alvarez-Erviti, L. et al. Delivery of siRNA to the mouse brain by systemic injection of targeted exosomes. Nat. Biotechnol. 29, 341-345 (2011). This study is the first to demonstrate exosomemediated siRNA delivery to the mouse brain

256. Kamerkar S et al. Exosomes facilitate therapeutic targeting of oncogenic KRAS in pancreatic cancer. Nature 546, 498-503 (2017).

257. Kordelas, L. et al. MSC-derived exosomes: a novel tool to treat therapy-refractory graft-versus-host disease. Leukemia 28, 970-973 (2014).

258. Lai, R. C. et al. Exosome secreted by MSC reduces myocardial ischemia/reperfusion injury. Stem Cell Res. 4, 214-222 (2010)

259. Giebel, B., Kordelas, L. \& Börger, V. Clinical potential of mesenchymal stem/stromal cell-derived extracellular vesicles. Stem Cell Investig. 4, 84 (2017)

260. Katakowski, M. et al. Exosomes from marrow stromal cells expressing miR-146b inhibit glioma growth. Cancer Lett. 335, 201-204 (2013)

261. Mendt, M. et al. Generation and testing of clinical-grade exosomes for pancreatic cancer. JCI Insight 3, e99263 (2018).

262. Lamichhane, T. N. et al. Oncogene knockdown via active loading of small RNAs into extracellular vesicles by sonication. Cell Mol. Bioeng. 9, 315-324 (2016).

263. Haraszti, R. A. et al. Optimized cholesterol-siRNA chemistry improves productive loading onto extracellular vesicles. Mol. Ther. 26, 1973-1982 (2018).

264. Didiot, M.-C. et al. Exosome-mediated delivery of hydrophobically modified siRNA for huntingtin mRNA silencing. Mol. Ther. 24, 1836-1847 (2016).

265. Gao, X. et al. Anchor peptide captures, targets, and loads exosomes of diverse origins for diagnostics and therapy. Sci. Transl Med. 10, eaat0195 (2018).

266. Cooper, J. M. et al. Systemic exosomal siRNA delivery reduced $\alpha$-synuclein aggregates in brains of transgenic mice. Mov. Disord. 29, 1476-1485 (2014).

267. Yang, J., Zhang, X., Chen, X., Wang, L. \& Yang, G. Exosome mediated delivery of miR-124 promotes neurogenesis after ischemia. Mol. Ther. Nucleic Acids 7, 278-287 (2017).

268. Ohno, S. et al. Systemically injected exosomes targeted to EGFR deliver antitumor microRNA to breast cancer cells. Mol. Ther. 21, 185-191 (2013)

269. Pi, F. et al. Nanoparticle orientation to control RNA loading and ligand display on extracellular vesicles for cancer regression. Nat Nanotechnol. 13, 82-89 (2018).

270. Nordin, J. Z. et al. Ultrafiltration with size-exclusion liquid chromatography for high yield isolation of extracellular vesicles preserving intact biophysical and functional properties. Nanomedicine 11, 879-883 (2015).

271. Kapadia, C. H., Melamed, J. R. \& Day, E. S. Spherical nucleic acid nanoparticles: therapeutic potential. BioDrugs 32, 297-309 (2018).

272. Jensen, S. A. et al. Spherical nucleic acid nanoparticle conjugates as an RNAi-based therapy for glioblastoma. Sci. Transl Med. 5, 209ra152 (2013).

273. Randeria, P. S. et al. siRNA-based spherical nucleic acids reverse impaired wound healing in diabetic mice by ganglioside $\mathrm{GM} 3$ synthase knockdown. Proc. Natl Acad. Sci. USA 112, 5573-5578 (2015).

274. Nemati, H. et al. Using siRNA-based spherical nucleic acid nanoparticle conjugates for gene regulation in psoriasis. J. Control. Rel. 268 , 259-268 (2017).

275. Lee, $\mathrm{H}$. et al. Molecularly self-assembled nucleic acid nanoparticles for targeted in vivo siRNA delivery. Nat. Nanotechnol. 7, 389-393 (2012).

276. Mohri, K. et al. Design and development of nanosized DNA assemblies in polypod-like structures as efficient vehicles for immunostimulatory $\mathrm{CpG}$ motifs to immune cells. ACS Nano 6, 5931-5940 (2012).

277. Jiang, D. et al. Efficient renal clearance of DNA tetrahedron nanoparticles enables quantitative evaluation of kidney function. Nano Res. 12 637-642 (2019)

278. Li, H. et al. siRNA suppression of hTERT using activatable cell-penetrating peptides in hepatoma cells. Biosci Rep 35, e00181 (2015).

279. Jiang, T. et al. Tumor imaging by means of proteolytic activation of cell-penetrating peptides. Proc. NatI Acad. Sci. USA 101, 17867-17872 (2004).

280. Rozema, D. B. et al. Dynamic polyconjugates for targeted in vivo delivery of siRNA to hepatocytes. Proc. Natl Acad. Sci. USA 104, 12982-12987 (2007).

281. Douglas, S. M., Bachelet, I. \& Church, G. M. A logic-gated nanorobot for targeted transport of molecular payloads. Science $335,831-834$ (2012).

282. Torchilin, V. P. Multifunctional, stimuli-sensitive nanoparticulate systems for drug delivery. Nat. Rev. Drug Disco. 13, 813-827 (2014).

283. Prasad, V. Nusinersen for spinal muscular atrophy: are we paying too much for too little? JAMA Pediatr. 172, 123-125 (2018)

284. Ng, E. W. M. et al. Pegaptanib, a targeted anti-VEGF aptamer for ocular vascular disease. Nat. Rev. Drug Discov. 5, 123-132 (2006).

285. Zhao, $\mathrm{X}$. et al. Mechanisms involved in the activation of C/EBP $\alpha$ by small activating RNA in hepatocellular carcinoma. Oncogene 38, 3446-3457 (2019).

286. Tuerk, C. \& Gold, L. Systematic evolution of ligands by exponential enrichment: RNA ligands to bacteriophage T4 DNA polymerase. Science 249, 505-510 (1990).

287. Robertson, D. L. \& Joyce, G. F. Selection in vitro of an RNA enzyme that specifically cleaves single-stranded DNA. Nature 344, 467-468 (1990).

288. Ellington, A. D. \& Szostak, J. W. In vitro selection of RNA molecules that bind specific ligands. Nature 346 818-822 (1990)

289. Burmeister, P. E. et al. Direct in vitro selection of a 2'-O-methyl aptamer to VEGF. Chem. Biol. 12, 25-33 (2005).

290. Ruckman, J. et al. 2'-Fluoropyrimidine RNA-based aptamers to the 165-amino acid form of vascular endothelial growth factor (VEGF165). Inhibition of receptor binding and VEGF-induced vascular permeability through interactions requiring the exon 7-encoded domain. J. Biol. Chem. 273, 20556-20567 (1998).

291. Eulberg, D. \& Klussmann, S. Spiegelmers: biostable aptamers. Chembiochem 4, 979-983 (2003).

292. Jinek, M. et al. A programmable dual-RNA-guided DNA endonuclease in adaptive bacterial immunity. Science 337, 816-821 (2012).

293. Cong, L. et al. Multiplex genome engineering using CRISPR/Cas systems. Science 339, 819-823 (2013).

294. Mali, P. et al. RNA-guided human genome engineering via Cas9. Science 339, 823-826 (2013).

295. Chavez, A. et al. Highly-efficient Cas9-mediated transcriptional programming. Nat. Methods 12, 326-328 (2015)

296. Kiani, S. et al. CRISPR transcriptional repression devices and layered circuits in mammalian cells. Nat. Methods 11, 723-726 (2014).

297. Ran, F. A. et al. In vivo genome editing using Staphylococcus aureus Cas9. Nature 520, 186-191 (2015).

298. Xu, X. et al. Delivery of CRISPR/Cas9 for therapeutic genome editing. J. Gene Med. 21, e3107 (2019).

299. Lee, K. et al. Nanoparticle delivery of Cas 9 ribonucleoprotein and donor DNA in vivo induces homology-directed DNA repair. Nat. Biomed. Eng. 1 , 889-901 (2017).

300. Zuris, J. A. et al. Cationic lipid-mediated delivery of proteins enables efficient protein-based genome editing in vitro and in vivo. Nat. Biotechnol. 33 , 73-80 (2015). 
301. Gao, X. et al. Treatment of autosomal dominant hearing loss by in vivo delivery of genome editing agents. Nature 553, 217-221 (2018).

302. Ramakrishna, S. et al. Gene disruption by cell-penetrating peptide-mediated delivery of Cas 9 protein and guide RNA. Genome Res. 24, 1020-1027 (2014).

\section{Acknowledgements}

The authors thank R. Raz for assistance with chemical structures.

\section{Author contributions}

T.C.R. and M.J.A.W. discussed content and wrote the article T.C.R., R.L. and M.J.A.W. revised the manuscript before submission; and T.C.R. developed all of the figures.

\section{Competing interests}

Complete details of relationships, compensated and uncompensated, for R.L. can be found in the Supplementary information. M.J.A.W. is a founder and shareholder of Evox Therapeutics and PepGen Ltd, companies dedicated to the commercialization of extracellular vesicle therapeutics and peptide-enhanced therapeutic oligonucleotide delivery, respectively. T.C.R. declares no competing financial interests.

\section{Peer review information}

Nature Reviews Drug Discovery thanks the anonymous reviewers for their contribution to the peer review of this work.

\section{Publisher's note}

Springer Nature remains neutral with regard to jurisdictional claims in published maps and institutional affiliations.
Supplementary information

Supplementary information is available for this paper at https://doi.org/10.1038/s41573-020-0075-7.

\section{RELATED LINKS}

n-Lorem Foundation: www.nlorem.org

Regulatory approval for viltolarsen in Japan: https://

www.nippon-shinyaku.co.jp/file/download.php?file_id=2965 Sarepta Therapeutics' pipeline: https://www.sarepta.com/

products-pipeline/pipeline

Stoke Therapeutics' science: https://www.stoketherapeutics. com/our-science/

(C) Springer Nature Limited 2020 